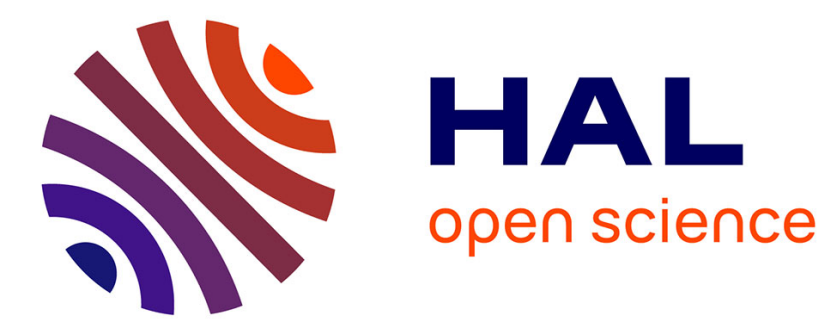

\title{
Rois et tyrans dans le théâtre d'Aristophane
}

Dominique Lenfant

\section{To cite this version:}

| Dominique Lenfant. Rois et tyrans dans le théâtre d'Aristophane. 2004. halshs-00001091

\section{HAL Id: halshs-00001091 https://shs.hal.science/halshs-00001091}

Preprint submitted on 30 Jan 2004

HAL is a multi-disciplinary open access archive for the deposit and dissemination of scientific research documents, whether they are published or not. The documents may come from teaching and research institutions in France or abroad, or from public or private research centers.
L'archive ouverte pluridisciplinaire HAL, est destinée au dépôt et à la diffusion de documents scientifiques de niveau recherche, publiés ou non, émanant des établissements d'enseignement et de recherche français ou étrangers, des laboratoires publics ou privés. 


\section{Article paru dans Ktèma 22, 1997}

\section{Rois et tyrans dans le théâtre d'Aristophane}

RÉSUMÉ. - La terminologie du pouvoir personnel présente chez Aristophane deux usages distincts selon que l'univers de référence est mythique ou contemporain. Les souverains mythiques sont indifféremment désignés par $\beta \alpha \sigma \imath \lambda \varepsilon u ́ \zeta$ et $\tau u ́ \rho \alpha v v o \zeta$, conformément à l'usage des tragédies de l'époque. Au contraire, les références au monde contemporain adoptent l'usage quotidien des termes au temps d'Aritophane et supposent une nette distinction entre le $\beta \alpha \sigma \imath \lambda \varepsilon u ́ \zeta$, souverain éloigné qu'est le Roi de Perse, et le $\tau u ́ \rho a v v o \zeta$, ennemi interne de la démocratie athénienne. Les diverses allusions à la tyrannie traduisent l'actualité politique et son interprétation par l'auteur comique, qui dénonce le galvaudage du terme par les démagogues dans les années 420 , tourne en dérision la phobie des tyrans après les affaires de 415 , mais renonce à en faire rire après les événements de 411.

ABSTRACT. - The terminology of personal power in Aristophanes' plays presents two different uses depending on whether the referent is mythical or contemporary. When the sovereign of the mythical world is equally called either $\beta \alpha \sigma \imath \lambda \varepsilon \cup ́ \varsigma$ or $\tau u ́ \rho \alpha v v o s$ (in accordance with contemporary tragical use), in the contemporary world $\beta \alpha \sigma \iota \lambda \varepsilon u ́ \zeta$ usually designates the Persian king and $\tau$ v́p $\alpha v v o \zeta$ the internal ennemy of Athenian democracy (in compliance with the daily use of that time). The different hints to tyranny express current political events and their interpretation by Aristophanes, who reproves the misuse of the term by demagogues in the 420 's, makes fun of the fear of tyrants after the events of 415 , but gives up laughing at that subject after the events of 411 .

Il peut sembler curieux et incongru de s'interroger sur les rois et les tyrans du théâtre d'Aristophane, dont le décor de prédilection est l'Athènes démocratique de son temps et dont les personnages, contrairement à ceux de la tragédie, ne sont qu'exceptionnellement des barbares $\left({ }^{1}\right)$. Mais, à défaut de paraître sur scène, rois et tyrans sont présents dans la bouche des personnages comiques, ce qui n'a rien pour étonner : pas plus que la démocratie athénienne qui lui sert de décor, l'univers comique ne pouvait rester muet sur les formes de régime personnel qui lui étaient si étrangères.

Pour définir le sens de telles allusions, il convient de s'attacher aux termes grecs qui désignent le détenteur d'un pouvoir personnel. Cela s'impose d'autant plus que l'usage n'en est pas univoque, comme il ressort de leur examen dans le théâtre d'Euripide : chez ce dernier, $\tau u ́ \rho \alpha v v o \zeta$, deux fois plus fréquent que $\beta \alpha \sigma \imath \lambda \varepsilon u ́ \zeta$, est affecté d'un potentiel négatif, qui conduit à le préférer quand il s'agit de critiquer le pouvoir ou d'en souligner le caractère redoutable, mais, dans

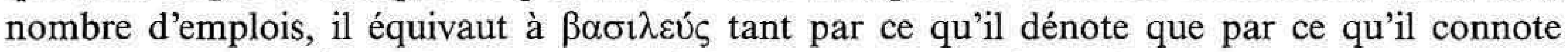
- ces deux valeurs interdisent donc de lui réserver une traduction unique, le contexte permettant seul de trancher entre «souverain» et «tyran» $\left({ }^{2}\right)$.

(1) Les personnages barbares des comédies conservées d'Aristophane sont presque toujours des esclaves. Le Pseudartabas des Acharniens s'avère n'être, tout comme les eunuques qui l'accompagnent, rien de plus qu'un Athénien déguisé (115 sq.). Sur les barbares dans la comédie grecque en général, cf. T. Long, Barbarians in Greek Comedy, Southern Illinois University, 1986.

Sur les barbares dans la tragédie, cf. H. H. BACon, Barbarians in Greek Tragedy, New Haven, 1961, et E. HALl, Inventing the Barbarian. Greek Self-Definition through Tragedy, Oxford, 1989.

(2) Je me permets de rappeler ici les conclusions de mon article sur «Le vocabulaire du pouvoir personnel chez Euripide», Ktèma 18, 1993, pp. 29-40. 
En fait, la terminologie d'Aristophane contraste étrangement avec celle de son contemporain Euripide et semble d'emblée moins confuse. Les termes essentiels pour désigner le détenteur

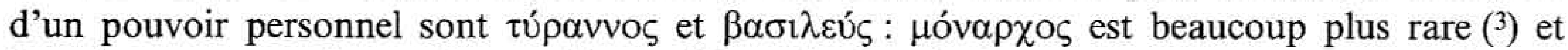

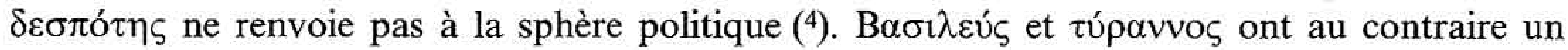
sens politique et l'examen des textes invite à distinguer deux sortes d'emplois en fonction du type de référent.

Quand le référent est une divinité ou un héros légendaire et qu'il relève de la sphère mythique, Aristophane emploie indifféremment $\beta \alpha \sigma \imath \lambda \varepsilon v ́ \varsigma$ et $\tau u ́ p \alpha v v o \zeta$, sans que ce dernier terme revête une valeur polémique particulière. Le premier peut se traduire par «roi», le second par «souverain».

Quand ils désignent des contemporains, les deux termes ont, au contraire, des référents et des connotations bien distincts: Aristophane est tributaire des emplois contemporains de

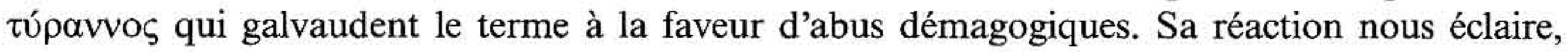
en outre, sur la manière dont furent perçus de son temps menace oligarchique et danger tyrannique.

\section{LE POUVOIR PERSONNEL DANS LA SPHÈRE MYTHIQUE}

B $\alpha \sigma i \lambda \varepsilon v ́ \varsigma$ et $\tau u ́ p \alpha v v o \varsigma$ désignent, dans la sphère mythique, les détenteurs d'un pouvoir personnel qui sont ou des dieux, ou des oiseaux, ou des héros.

\section{A) Les dieux}

La souveraineté de telle divinité, censée s'exercer soit sur les autres dieux, soit sur telle partie du monde ou tel champ humain, s'exprime généralement par des mots de la famille de $\beta \alpha \sigma \imath \lambda \varepsilon v ́ c$. Ainsi, Zeus, en tant que souverain des dieux et des hommes, est qualifié le plus

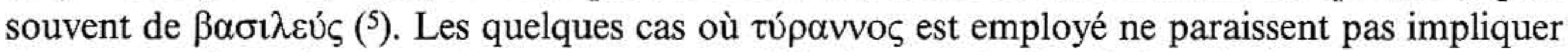
de connotation négative et le terme est alors l'équivalent de $\beta a \sigma l \lambda \varepsilon v ́ \varsigma$ : ainsi, quand le chœur

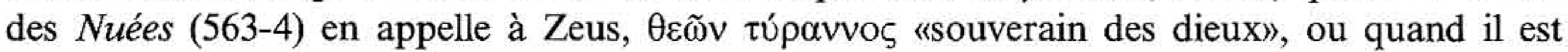
question de la topavvís de Poséidon (Oiseaux 1605) ou de celle de Zeus (Oiseaux 1643, Ploutos 124).

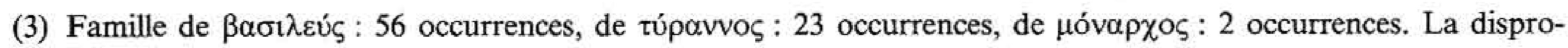

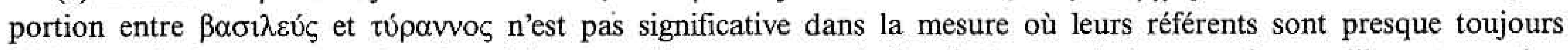
distincts. Ainsi trouve-t-on dans les Acharniens 11 occurrences de $\beta \alpha \sigma \iota \lambda \varepsilon v ́ \varsigma$ pour la bonne raison qu'il y est question du Grand Roi.

(4) $\Delta \varepsilon \sigma \pi o ́ \tau \eta \zeta$ désigne soit un dieu, soit, dans les trois quarts des cas, le maître d'un esclave. On ne recense aucun exemple où il ait, comme chez Hérodote, un sens politique. Il se cantonne donc dans le registre domestique ou religieux, comme dans le théâtre d'Euripide, chez qui l'on avait cependant observé des dérogations significatives, les emplois politiques du terme désignant un pouvoir jugé excessif (art. cit. pp. 29-30).

(5) Dans la plupart des cas, $\beta \alpha \sigma \iota \lambda \varepsilon v ́ \zeta$ apparaît en tant qu'épiclèse dans l'apostrophe $\tilde{\omega}$ Zzṽ $\beta \alpha \sigma \imath \lambda \varepsilon v ̃$ (Nuées 2, 153, Guêpes 624, Oiseaux 223, Grenouilles 1278, Ploutos 1095). Toutefois Oiseaux 514, 515, 610 évoquent Zeus comme

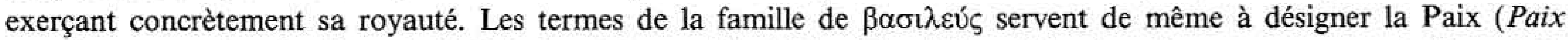

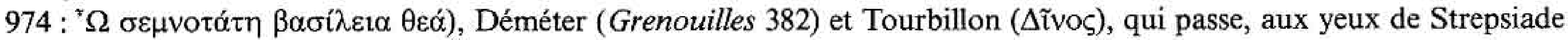
et suivant l'enseignement du Socrate aristophanesque, pour régner après avoir chassé Zeus (Nuées, 381, 828, 1471).

$\Pi \alpha \mu \beta \alpha \sigma i \lambda \varepsilon i \alpha$ ureine absolue» apparait aussi par deux fois dans les Nuées (357: apostrophe aux Nuées; 1150 : à Fourberie). 
B) Du côté des oiseaux, censés avoir régné avant les dieux (Oiseaux 482), donc assimilables à des divinités, le constat est logiquement le même, avec cependant un cas qui peut faire hésiter.

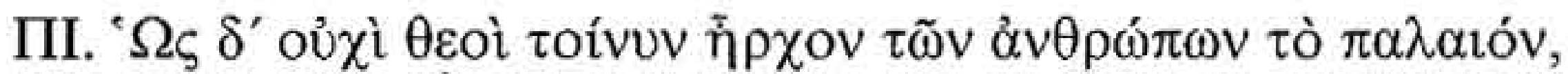

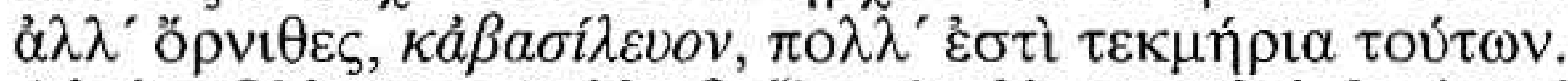

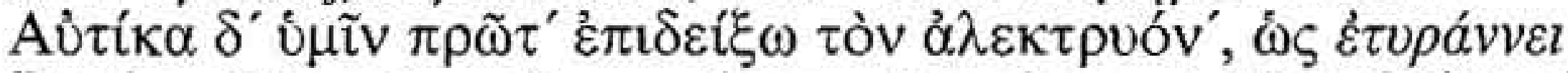

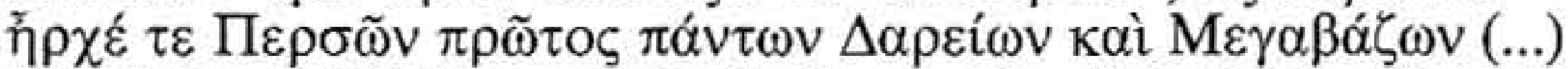

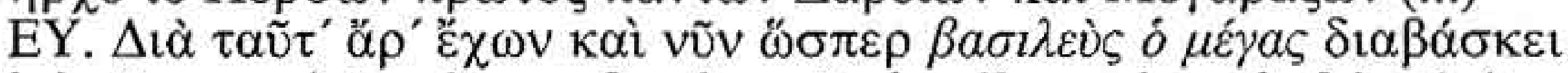

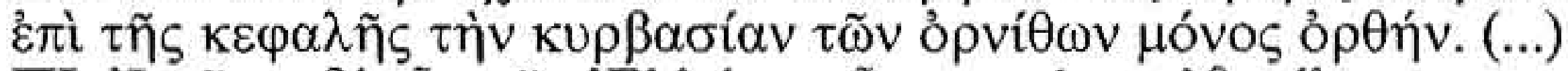

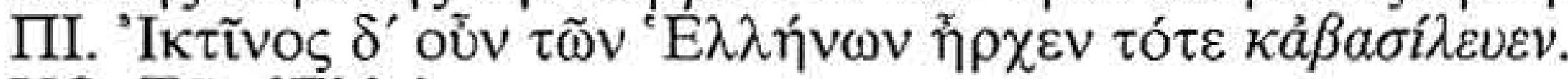

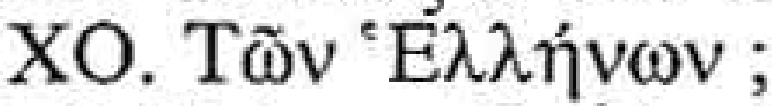

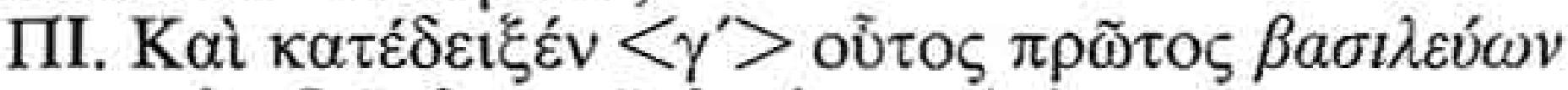

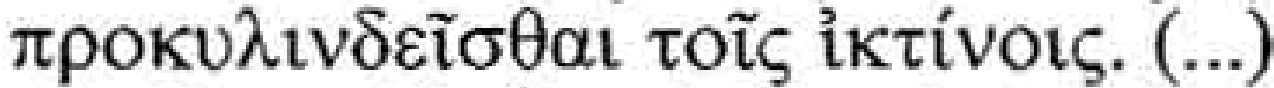

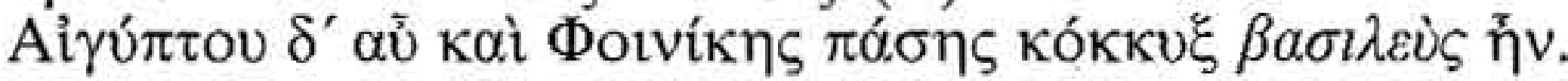

«Pisthétaire. - Ce n'étaient pas les dieux qui commandaient jadis aux hommes et exerçaient la royauté, mais les oiseaux, les preuves en abondent. Et, tout de suite, je vous citerai en premier lieu le coq, qui exerçait la souveraineté (ou: la tyrannie) et commandait aux Perses, premier de tous les Darius et Mégabaze (...).

Evelpide. - Voilà donc pourquoi il se pavane maintenant encore comme le Grand Roi, seul des oiseaux à porter sur la tête la tiare droite (...).

Pisthétaire. - Le milan commandait alors aux Grecs et exerçait la royauté.

Coryphée. - Aux Grecs?

Pisthétaire. - Oui. Et c'est lui qui, sous son règne, leur enseigna le premier à se rouler à terre devant les milans (...).

Le coucou était roi d'Egypte et de toute la Phénicie» (6).

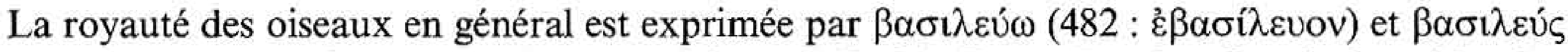
(o $\mu \varepsilon \gamma_{\gamma} \alpha \varsigma$ ) est l'expression consacrée pour désigner le Roi de Perse. De plus, $\beta \alpha \sigma \iota \lambda \varepsilon u ́ s$ prédomine nettement pour désigner les souverains des différentes parties du monde. Ce n'est peut-être pas un hasard si le verbe tupavvõ est réservé aux Perses, que les Athéniens présentaient volontiers comme leur opposé politique $\left(^{7}\right)$. Peut-être est-ce en raison du potentiel négatif du terme, qui est généralement insensible dans la sphère mythique, mais le cas présent ne faitil pas précisément interférer la sphère politique contemporaine? Le roi de Perse ne relève pas du mythe. Toutefois, la nuance est incertaine et il n'y a pas lieu d'exagérer la différence

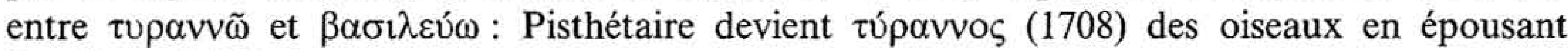
B $\alpha \sigma i ́ \lambda \varepsilon ı \alpha(1536-7)$.

C) S'agissant des rois légendaires, c'est encore $\beta \alpha \sigma \imath \lambda \varepsilon v ́ \varsigma$ qui prédomine, aussi bien pour Agamemnon ou Ménélas (Oiseaux 509) que pour les rois de la tragédie (Grenouilles 1063). Le seul exemple conservé d'emploi de túpavvo̧ se trouve dans un fragment des Lemniennes:

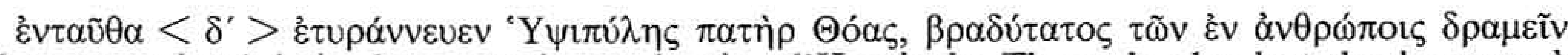
«La souveraineté était alors exercée par le père d'Hypsipyle, Thoas, le plus lent des hommes à la course» $\left({ }^{8}\right)$.

Le terme n'a probablement pas de valeur polémique et l'on peut sans doute suivre Ammonius, qui nous transmet cette citation dans son ouvrage sur les différences entre termes voisins $\left({ }^{9}\right)$,

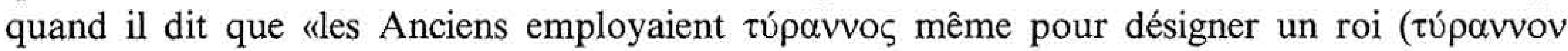

(6) Oiseaux 481-484, 486-487, 499-501, 504.

(7) Il est cependant exceptionnel qu'un roi de Perse soit qualifié de túpavvoç. Voir, à propos de l'usage hérodotéen, E. Lêvy, «Basileus et turannos chez Hérodote», Ktèma 18, 1993, p. 10.

(8) F373 Kassel-Austin (= 357 Kock).

(9) Ammonius, De adfinium vocabulorum differentia, éd. K1. Nickau, Leipzig, 1966. 


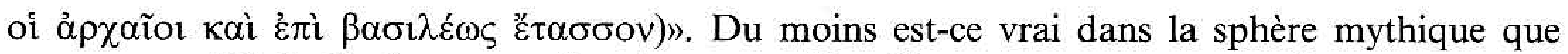
nous avons définie. Il n'en va pas de même quand le référent est un contemporain.

\section{LE POUVOIR PERSONNEL DANS LE MONDE CONTEMPORAIN}

Dans le monde contemporain, $\beta \alpha \sigma \imath \lambda \varepsilon v ́ \varsigma$ et $\tau u ́ p \alpha v v o \zeta$ ont des emplois nettement distincts : ils n'ont ni les mêmes référents ni les mêmes connotations.

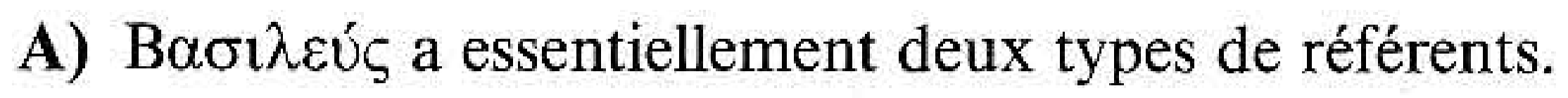

1) Le premier n'a rien pour surprendre: $\beta \alpha \sigma \imath \lambda \varepsilon v ́ \varsigma$ ou $\beta \alpha \sigma \imath \lambda \varepsilon v ́ \varsigma$ ó $\mu \varepsilon ́ \gamma \alpha \varsigma$ désigne, comme il est usuel, le Roi de Perse $\left({ }^{10}\right)$, et son évocation est associée aux idées d'éloignement spatial ( $\left.{ }^{11}\right)$, d'orgueil $\left({ }^{12}\right)$, de privilèges exclusifs $\left({ }^{13}\right)$ et de richesse colossale $\left({ }^{14}\right)$. C'est aussi celui avec qui l'on est accusé de comploter, comme en témoignent les accusations de médisme proférées par le Paphlagonien contre le Charcutier ( $\left.{ }^{15}\right)$.

2) B $\alpha \sigma i \lambda \varepsilon v ́ \varsigma$ sert aussi, de façon moins attendue, à désigner des personnages ou entités de l'actualité athénienne. On mettra à part le cas où ó $\beta \alpha \sigma \imath \lambda \varepsilon v ́ \varsigma$ est le terme technique qui désigne l'archonte-roi $\left(^{16}\right)$, pour s'arrêter sur deux emplois plus originaux, qui se réfèrent au pouvoir du peuple athénien, d'une part dans le cadre des tribunaux populaires, d'autre part à l'extérieur de ses frontières.

a) Le premier exemple est tiré des Guêpes (546-549) : s'apprêtant à vanter les délices de la condition de juge, Philocléon présente le pouvoir des héliastes comme une $\beta \alpha \sigma \imath \lambda \varepsilon i ́ \alpha$, une royauté :

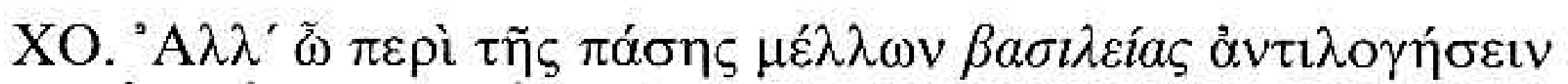

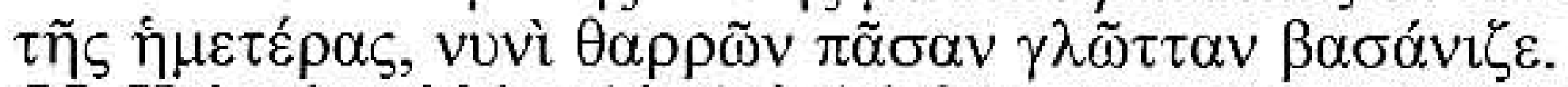

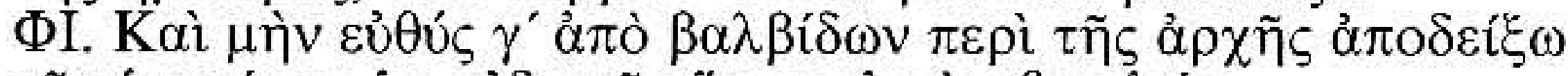

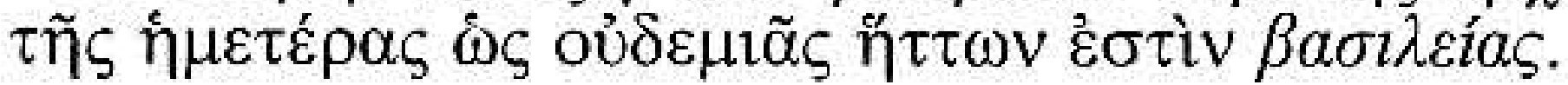

"Coryphée - Allons, toi qui t'apprêtes à répondre au sujet de la royauté totale qui est la nôtre, maintenant, hardi, mets à l'épreuve ta langue tout entière.

Philocléon - Eh bien, tout de suite, dès la borne de départ, je vais montrer que notre pouvoir ne le cède à aucune royauté».

En un sens, l'idée n'est pas entièrement originale, dans la mesure où l'idéologie démocratique aime à présenter le peuple comme souverain. Mais on évite généralement de qualifier le $\delta \tilde{\eta} \mu \circ \zeta$ de $\beta \alpha \sigma \imath \lambda \varepsilon v ́ \varsigma\left({ }^{17}\right)$. S’il est possible qu'Aristophane s'inspire ici d'un slogan démocratique, il n'en

(10) Acharniens (12 emplois, à propos de l'Oeil du Roi et des ambassadeurs censés être allés trouver le Grand Roi), Cavaliers 478, Oiseaux 486, Ploutos 170.

(11) Acharniens 647.

(12) Il se pavane comme le coq (Oiseaux 486) et tire fierté de sa richesse (Ploutos 170).

(13) Il est seul à pouvoir porter la tiare droite (Oiseaux 487).

(14) Ploutos 170, Acharniens 102.

(15) Il déclare qu'il va dénoncer au Conseil «tout ce que vous complotez avec les Mèdes et le Roi» ( $\pi \alpha v^{\prime} \theta^{\prime} \alpha$

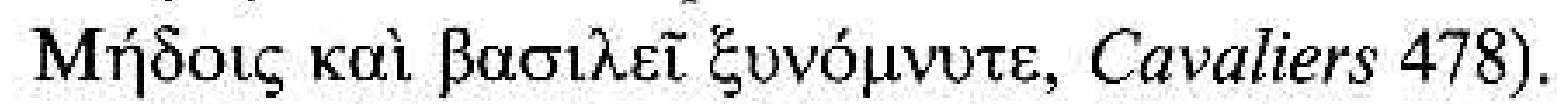

(16) Acharniens 1224 : c'est dans le cadre des monologues croisés de Dicéopolis et de Lamachos que le premier

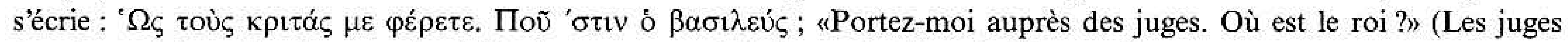
devaient arbitrer, l'archonte-roi présider les concours de boisson des fêtes de Dionysos).

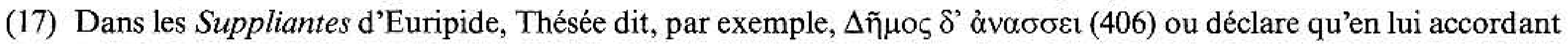


subvertit pas moins le sens de ce dernier à des fins critiques et comiques : il présente les juges comme motivés par la soif de pouvoir et la caricature qui sous-tend ensuite le développement, loin de mettre l'accent sur la souveraineté populaire, souligne l'arbitraire et l'injustice des héliastes et scinde le peuple en accusés sujets et en juges souverains.

b) Le second exemple se réfère au pouvoir du peuple athénien à l'extérieur de ses frontières : il s'agit du pouvoir promis par le Paphlagonien à Démos, lorsque, dans les Cavaliers (108687), Charcutier et Paphlagonien rivalisent d'oracles pour le séduire.

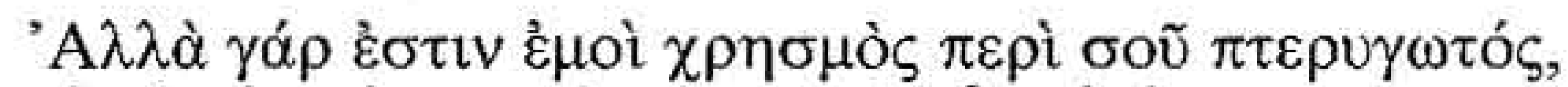

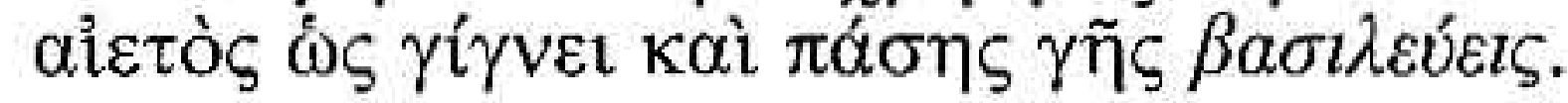

«Mais moi j'ai à ton sujet un oracle ailé où tu deviens un aigle et règnes sur toute la terre».

Comme on voit, il s'agit du pouvoir d'Athènes hors de ses frontières, de l'empire qu'elle y a ou y pourrait avoir et que suggèrent généralement le verbe $\alpha \propto \rho \chi \omega$ et le substantif $\alpha \rho \chi \eta ́ ~\left({ }^{18}\right)$ : peu auparavant, le Paphlagonien prédit ainsi à Démos qu'il commandera à toute la terre (äp $\xi_{\alpha}$

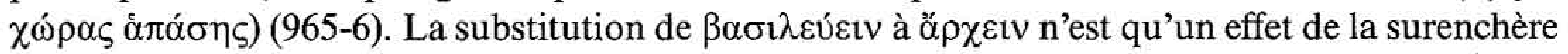
à laquelle se livrent Charcutier et Paphlagonien pour séduire Démos : le terme a une connotation plus prestigieuse, propice à flatter le peuple, et il n'a pas la valeur choquante de túpavvos, couramment employé dans les discours de Thucydide pour afficher la vraie nature du pouvoir d'Athènes sur ses alliés $\left.{ }^{(19}\right)$. En même temps, il est clair que son emploi par Aristophane prend une valeur satirique relative à l'impérialisme athénien et à la manière dont les démagogues en jouent ${ }^{(20)}$. Songeons à Alcibiade qui, dans le discours que Thucydide lui prête à la veille du départ de l'expédition de Sicile, déclare qu'après avoir annexé l'ìle à leur empire les Athéniens

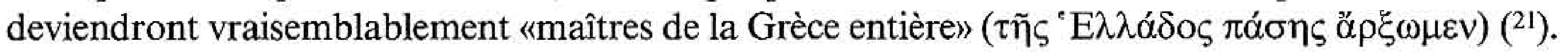

Ainsi, les deux exemples analysés se réfèrent à des représentations dues à des "démagogues» tels que Cléon et amplifiées par le poète à des fins comiques et satiriques. Citons, pour finir, un cas légèrement différent, l'apostrophe du coryphée des Cavaliers à Démos régénéré :

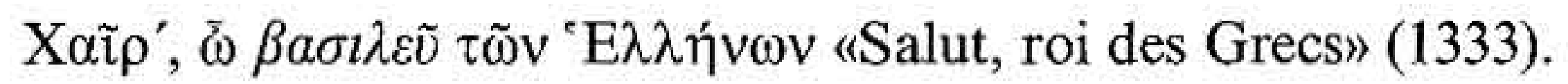

Il ne s'agit pas d'une référence à l'Empire, puisqu'il est question de faire revivre le Démos du bon vieux temps, de l'époque d'Aristide et de Miltiade. On y verra plutôt une allusion au prestige d'Athènes dans le monde grec au temps glorieux des guerres médiques, sans oublier, bien sûr, le comique qui ressort de la paradoxale assimilation du peuple athénien à un roi et du caractère parfaitement fictif d'une royauté exercée sur les Grecs (22).

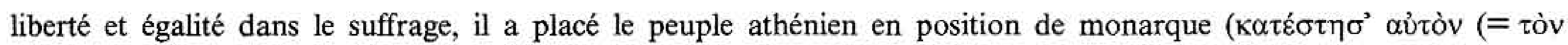

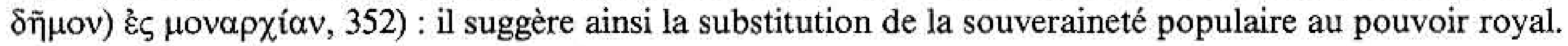

(18) Voir, par exemple, Guêpes 672 et 700.

(19) Cf. Thucydide I 122,3; 124,3; II 63,2 ; III 37,3. Voir, en outre, les références indiquées par H. BERvE, Die Tyrannis bei den Griechen, Munich, 1967, II, p. 628 «Athen als Tyran der Bündner».

(20) Ainsi, quand le Paphlagonien prétend qu'il est partisan de la guerre parce qu'il veut assurer à Démos

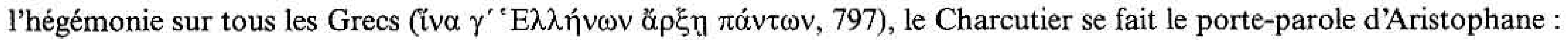

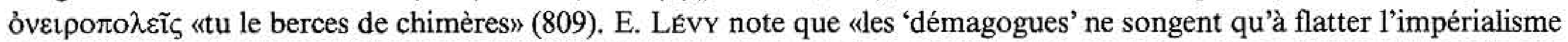
des masses» en cette période marquée par «l'apogée de l'idéologie de la puissance» (Athènes devant la défaite de 404, Paris, 1976, p. 122).

(21) Thucydide, VI,18. Certes, ce discours est censé dater de 415 , soit quelque dix ans après la représentation des Cavaliers en 424, mais, s'il n'a pu inspirer Aristophane, il est certain qu'il s'inscrit dans une pratique démagogique courante à l'époque. On songe, bien sûr, à Cléon, que caricature le personnage du Paphlagonien.

(22) Le même raisonnement vaut pour $\mu$ óv $\alpha \rho \chi \chi_{\text {S }}$ : trois vers plus haut (Cavaliers 1330), le même coryphée désigne 
Ainsi, $\beta \alpha \sigma \imath \lambda \varepsilon v ́ \varsigma$ n'a pas, en général, de valeur polémique. Si l'on excepte l'emploi technique du terme désignant l'archonte-roi, il renvoie à un pouvoir éloigné dans l'espace (Grand Roi), dans le temps ou dans l'imaginaire (héros, dieux, oiseaux). Les exceptions sont significatives : attribuer au peuple athénien une royauté dans les tribunaux ou à l'extérieur de ses frontières revient à dénoncer un abus de pouvoir. Enfin, $\beta \alpha \sigma \imath \lambda \varepsilon u ́ c$ et sa famille ne servent jamais à désigner une forme de pouvoir concurrente de la démocratie athénienne ou susceptible de la mettre en danger. Il en va différemment pour zúpavvos.

\section{B) $\tau u ́ \rho a v v o \zeta$ dans le monde contemporain}

Quand il s'applique aux hommes de l'époque contemporaine, à l'inverse de ce qui se passe quand il désigne les dieux, túpavvos a une connotation fortement négative et une valeur polémique marquée.

\section{1) Caractéristiques du tyran}

Les emplois du terme permettent de définir une image du tyran bien distincte de celle du $\beta \alpha \sigma \imath \lambda \varepsilon v$ c. Il renvoie à une forme spécifique de pouvoir, dont la référence historique est la tyrannie de l'Athènes archaïque, celle des Pisistratides, ainsi que l'attestent plusieurs références à Hippias ( $\left.{ }^{23}\right)$.

a) Tout d'abord, le tyran exerce un pouvoir exclusif et n'applique pas les lois de la cité, comme il ressort de ce passage des Guêpes (463-470) où le chœur des héliastes s'indigne d'être chassé à coups de bâton par les serviteurs de Bdélycléon - lequel, comme chacun sait, veut empêcher son père d'aller juger.

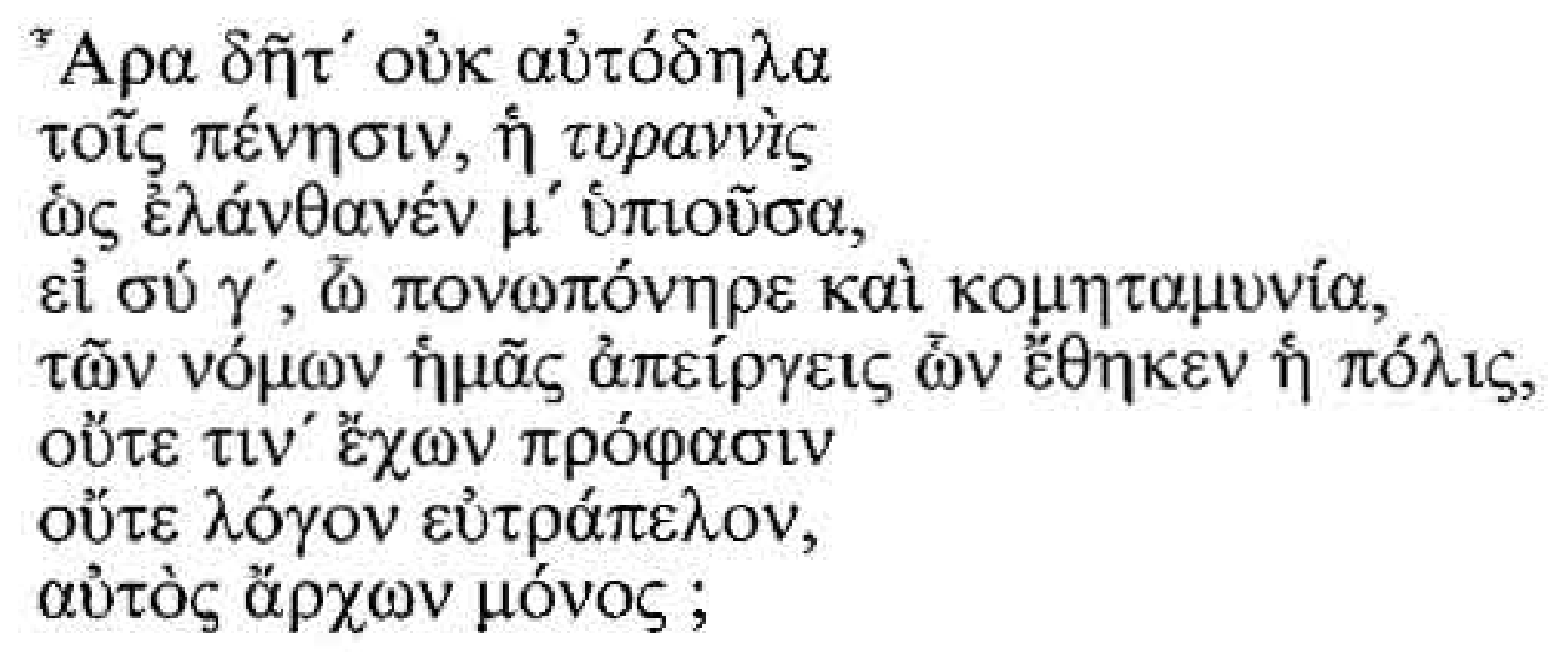

«Ah, ça ! Ne saute-t-il pas aux yeux des pauvres que c'est la tyrannie qui s'insinuait à mon insu, si te voilà, triple coquin, Amynias-à-tignasse, qui nous écartes des lois établies par la cité sans user de prétexte ni de raison spécieuse, te réservant le commandement pour toi seul ?»

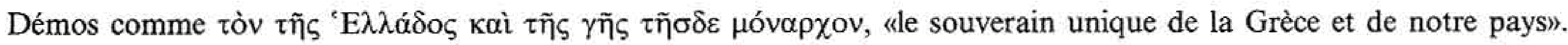

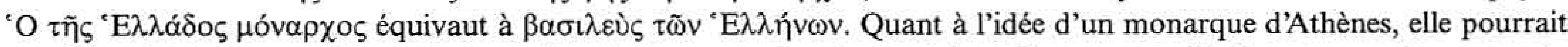
se référer à une expression contemporaine de la souveraineté populaire. Cf. supra n. 17. Les termes de $\mu$ ovapxía et de $\mu$ óv $\alpha \rho \chi \propto \zeta$ sont rares dans les textes de l'époque. On n'en trouve que six occurrences chez Euripide si l'on tient compte du Rhésos et des fragments, et seulement trois dans le cas contraire. Chez Aristophane, mis à part

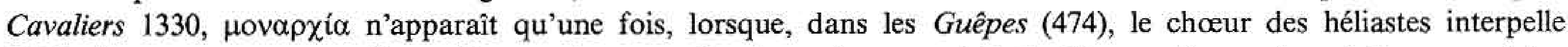

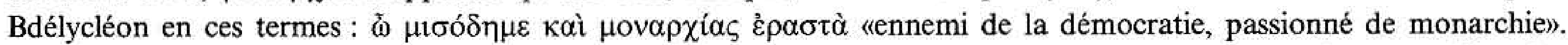
Le terme revêt ici une valeur polémique sans équivoque et semble relever du même registre que túpavvos. Cf. infra, à propos des vers 464 sq. et $486 \mathrm{sq}$.

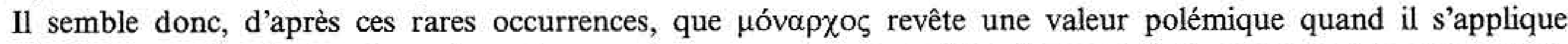
à un individu, mettant alors l'accent sur le pouvoir personnel en ce qu'il a d'injuste et de contraire à la démocratie, tandis que, s'il désigne le peuple tout entier, il se réfère, au contraire, à la souveraineté populaire, en tant que substitut du pouvoir personnel.

(23) Lysistrata 618, Guêpes 502, Oiseaux 1074-75 («l'un des tyrans morts»). 
La tyrannie est ici caractérisée par la non-application injustifiée des lois établies par la cité

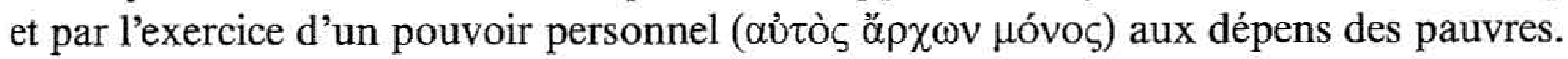

b) Le tyran détient le pouvoir de contraindre. Quand, dans les Guêpes (417), Bdélycléon refuse de lâcher son père, qu'il veut empêcher d'aller juger, le coryphée s'exclame :

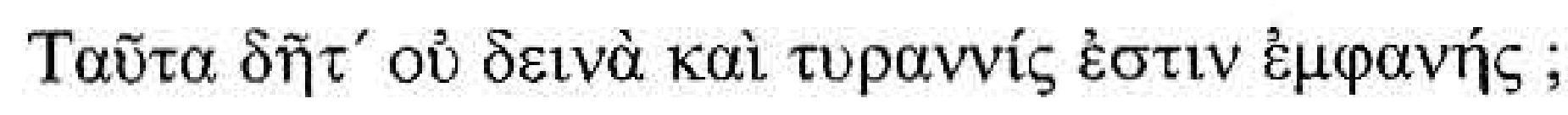

«Ah, ça! N'est-ce pas un comble, une tyrannie manifeste ?»

La tyrannie est ainsi associée au pouvoir de contrainte, mais aussi aux atteintes portées à cette pratique éminemment démocratique qu'est l'exercice de la justice dans le cadre de tribunaux populaires $\left({ }^{24}\right)$.

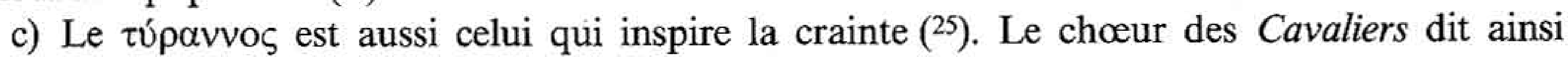

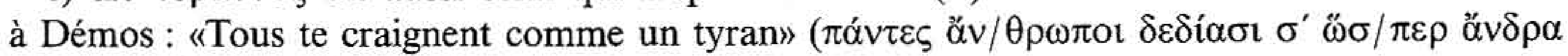

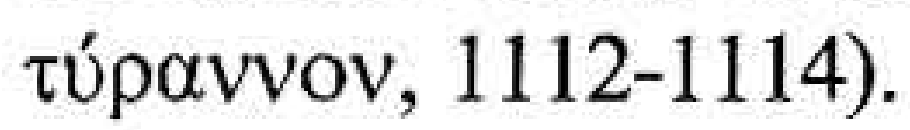

\section{2) Un terme à usage polémique}

Túpavvos sert plus à dénoncer qu'à désigner: le chœur des Guêpes accuse sans cesse Bdélycléon d'aspirer à la tyrannie (26); et, dans Lysistrata, le chœur des vieillards soupçonne les femmes d'agir «en vue de la tyrannie» (27). En effet, le túpavvos est volontiers associé à

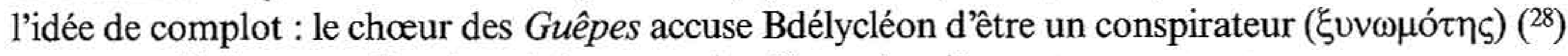
autant qu'il l'accuse d'aspirer à la tyrannie. Cette dernière est avant tout une menace pour la démocratie et peut à ce titre s'associer ou se mêler à d'autres formes de dangers, comme le médisme, le laconisme ou l'oligarchie.

\section{a) Médisme}

L'imprécation que, dans les Thesmophories (335 sq.), l'une des femmes prononce contre les hors-la-loi et les traîtres à la patrie, commence comme suit :

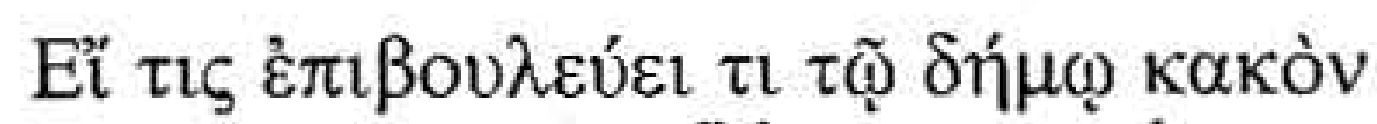

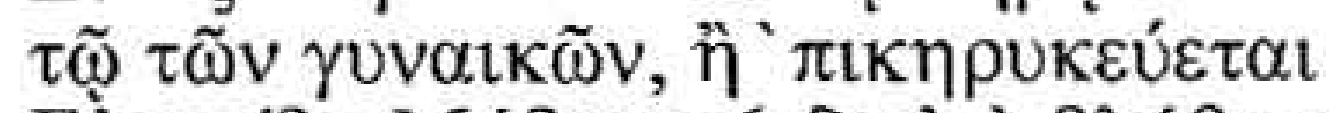

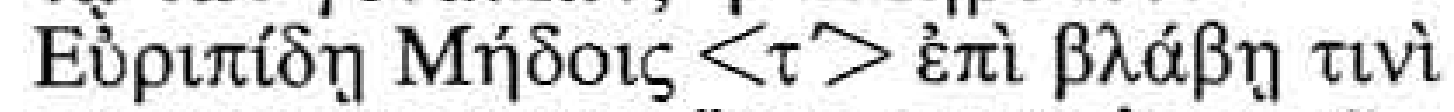

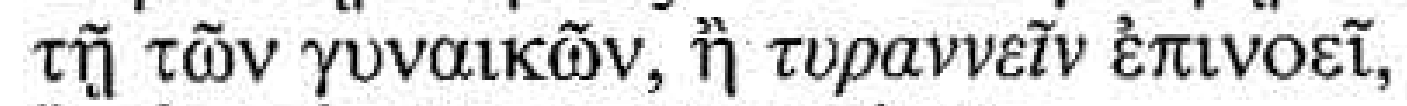

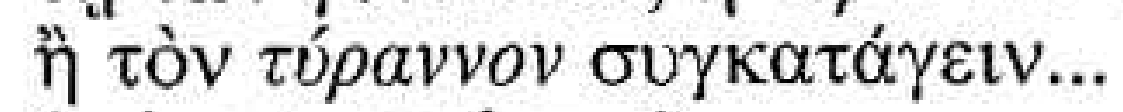

(suivent neuf vers)

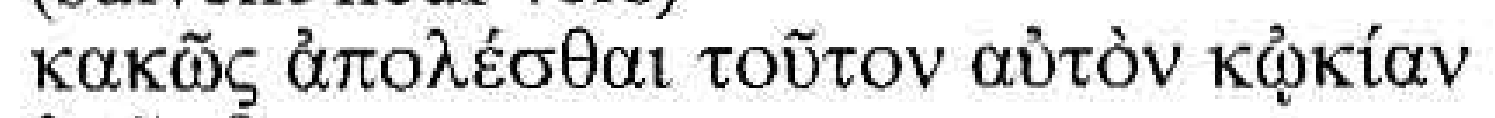

$\dot{\alpha} \rho \tilde{\alpha} \sigma \theta \varepsilon \ldots$

«Pour tout homme qui trame quelque mauvais coup contre le peuple... des femmes ou négocie avec Euripide et les Mèdes pour faire quelque tort aux femmes, pour tout homme qui songe à être tyran ou à aider au retour du tyran... priez qu'il périsse misérablement, lui et sa famille».

Aristophane parodie ici la pratique usuelle des malédictions solennelles qui précédaient l'ouverture des séances de l'Assemblée $\left({ }^{29}\right)$. Même si certaines de ces imprécations sont des inventions comiques en faveur des femmes - c'est évident pour celle qui vise Euripide (337)

(24) Cf. Guêpes 409-414: le chœur envoie les enfants appeler Cléon à venir combattre l'ennemi de l'Etat qui prétend qu'il ne faut pas juger de procès.

(25) Cette connotation est également courante chez Euripide. Cf. D. Lenfant, art. cit. (n. 1), pp. 32-38.

(26) Cf. infra, p. 195.

(27) Cf. infra, p: 193.

(28) Guêpes 345, 483 (cf. 488, 507).

(29) Sur cette pratique, cf. P. J. Rhodes, The Athenian Boule, Oxford, 1972, pp. 36-37. 
ou les séducteurs qui ne tiennent pas leurs promesses (343-4), celle qui concerne les tyrans reflète, quant à elle, un usage réel : on trouve une formule très proche dans le décret de Démophantos, d'après lequel les Athéniens devaient prêter serment de faire périr «quiconque

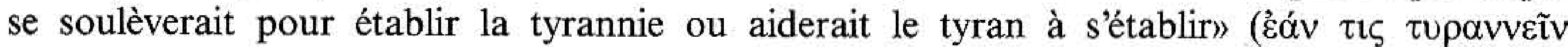

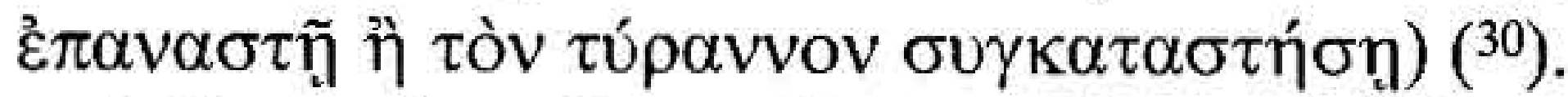

Ailleurs, la même pièce se fait probablement l'écho d'un slogan officiel qui présentait la tyrannie comme le repoussoir de la démocratie athénienne. Appelant Athéna, le chœur déclame :

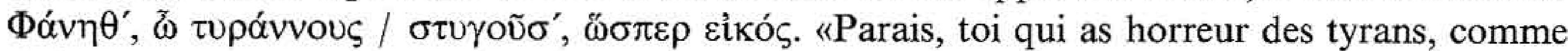
de juste» (Thesmophories 1143-4). Athéna symbolisait l'Athènes démocratique et c'est, du reste, lors des Panathénées qu'avait eu lieu l'assassinat d'Hipparque ( $\left.{ }^{31}\right)$. Quant à l'association du complot tyrannique au médisme (337-339), elle pourrait remonter à la tentative d'Hippias pour reprendre le pouvoir grâce aux Perses lors de la première guerre médique.

\section{b) Laconisme}

De même qu'au médisme, l'aspiration à la tyrannie est volontiers associée au laconisme. Ainsi, dans ce fragment des Paysans $\left({ }^{32}\right)$ :

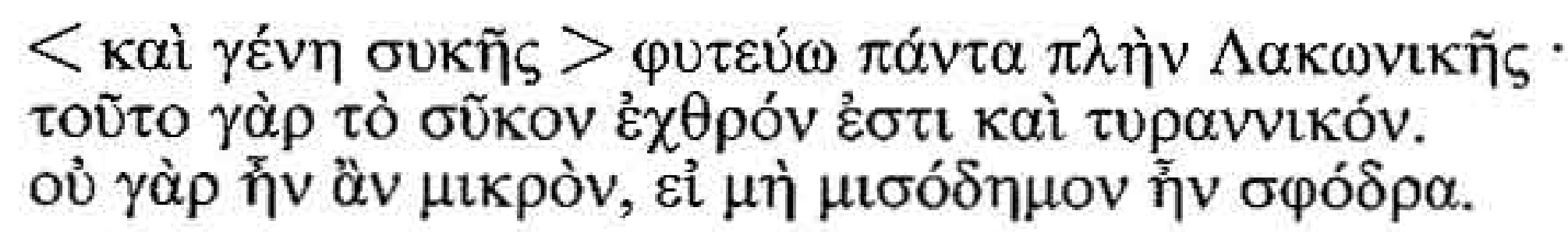

«je plante toutes les espèces de figuiers ${ }^{(33)}$ sauf le laconien, car cette figue est ennemie et de caractère tyrannique. En effet, elle ne serait pas petite si elle n'était pas franchement hostile au peuple».

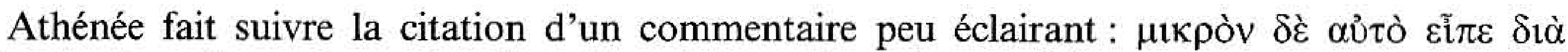

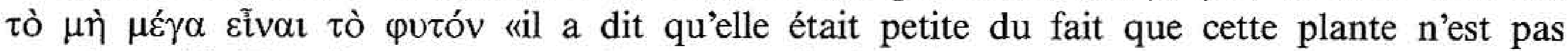
grande»... ( ${ }^{(34)}$ Faut-il supposer que l'espèce de figuier dite laconienne ( ${ }^{35}$ ) donne des figues de petite taille, donc moins nourrissantes que les autres - ce qui fait dire qu'elle est hostile au peuple ? Mais la perplexité visible d'Athénée, qui devait pourtant disposer du contexte, suggère

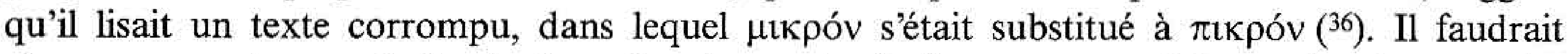
alors comprendre que le figuier laconien donne des fruits amers, qualité paradoxale pour des figues, généralement vantées pour leur saveur douce, et le sens figuré de $\pi$ ıкó́ favoriserait alors le jeu de mots. Quoi qu'il en soit, le sens global est clair et Sparte se trouve ainsi associée à l'idée de tyrannie et d'hostilité au régime démocratique.

(30) Décret cité par Andocide, Mystères, §96-98 (formule du serment: §97). Cf. IG I2, 304. Daté de juilletaoût 410 et consécutif à la chute des Quatre-Cents, il est donc postérieur aux Thesmophories, mais il ne fait que reprendre une formule ancienne. cf. M. Ostwald, "The Athenian Legislation against Tyranny and Subversion», TAPA $86,1955,103-28$, qui rassemble et discute les sources sur les mesures visant à contrer les tentatives de révolution tyrannique, et P.J. Rhodes, A Commentary on the Aristotelian Athenaion Politeia, Oxford, 1981, pp. 220-222.

(31) Aux Panathénées de 514. Cf. Hérodote, V,56 ; scolion de Callistratos apud Athénée, Deipnosophistes, XV,693; Thucydide, VI,56-57 ; Aristote, Constitution des Athéniens, XVIII.

Ajoutons que, sur plusieurs amphores panathénaïques (probablement postérieures à 403 av. J. C.), la statue des tyrannoctones figurait sur l'épisème du bouclier de la déesse. Cf. S. BRUNNSÂKER, The Tyrant-Slayers of Kritios and Nesiotes. A critical Study of the Sources and Restorations, Stockholm, 1971, pp. 104-105 et pl. 23.6.

(32) Poetae comici graeci, III,2, éd. R. Kassel \& C. Austin, F110 (108 Kock = Athénée III p. 75a).

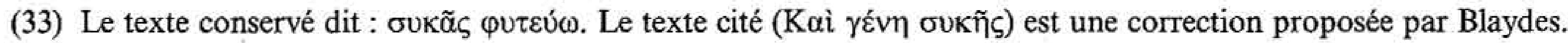

(34) L'évocation de la figue peut appeler celle du sycophante (comme dans Guêpes, 145 et 897, Ploutos 946), mais ce n'est pas le cas ici : planter, cueillir, manger des figues symbolise généralement chez Aristophane les plaisirs de la paix. Cf. Paix 558, où le chœur déclare vouloir saluer ses vignes et les figuiers qu'il a plantés dans sa jeunesse

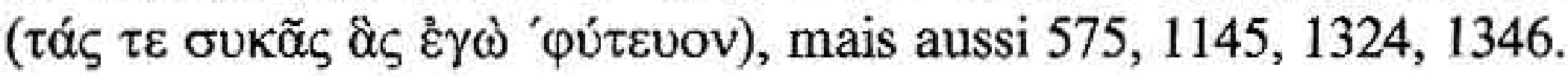

(35) Voir R.E. VI,2 p. 2123.

(36) Je dois cette suggestion à Anne Jacquemin. 
La chose est plus nette encore dans ce passage de Lysistrata (616-635) où, les femmes ayant annoncé leur décision de faire la grève de l'amour tant que les hommes n'auraient pas conclu la paix, le chœur des vieillards athéniens réplique ainsi :

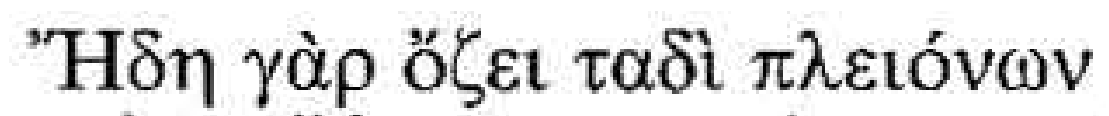

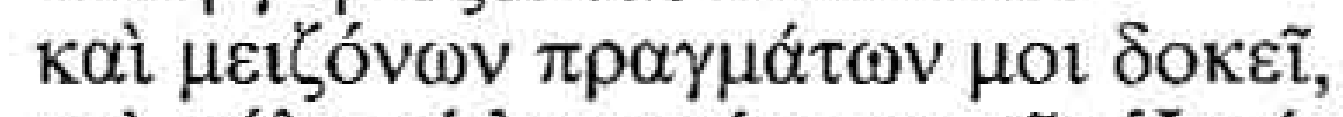

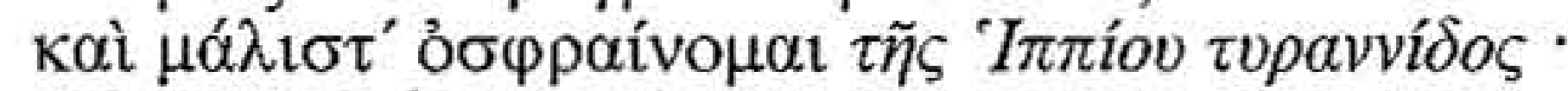

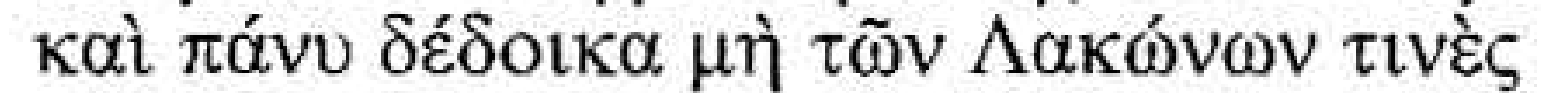

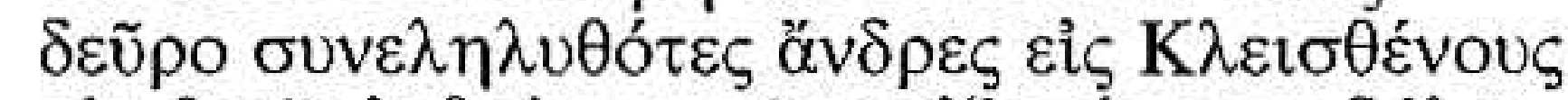

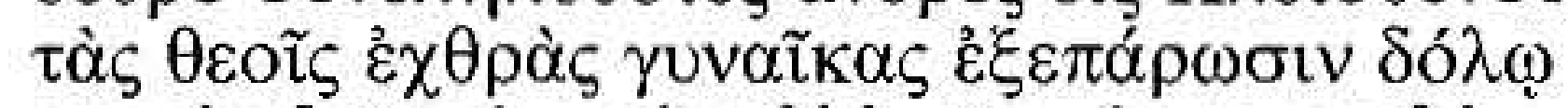

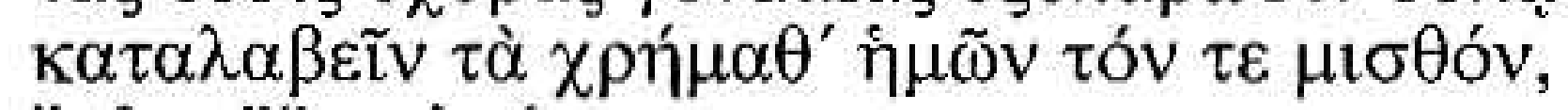

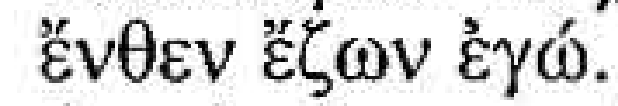

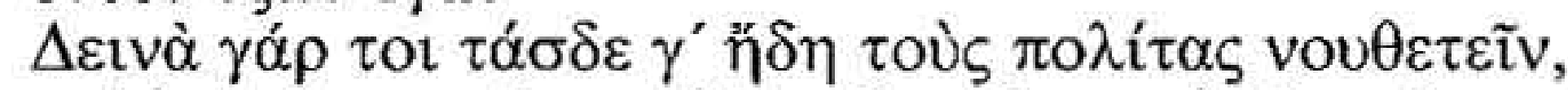

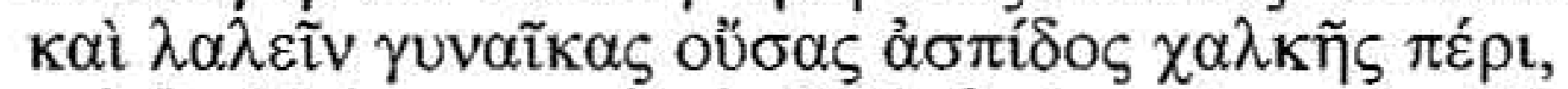

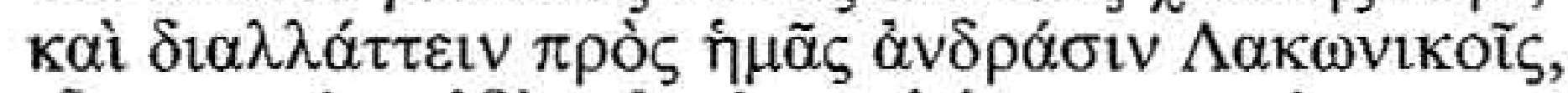

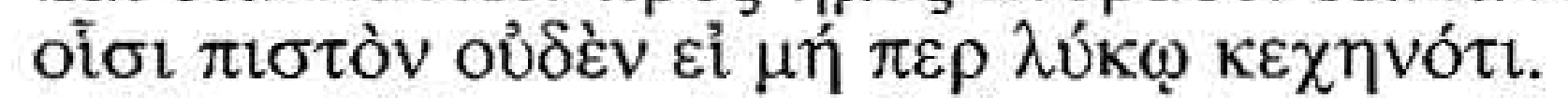

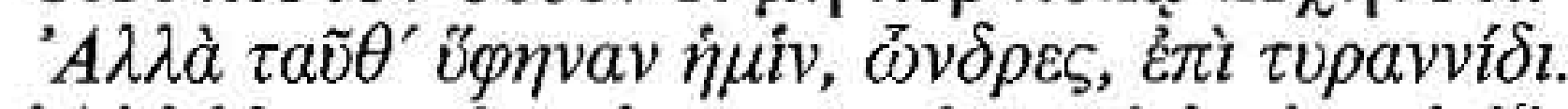

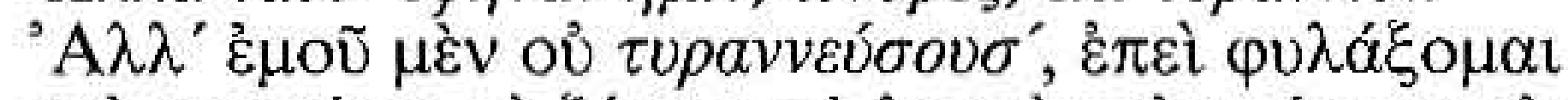

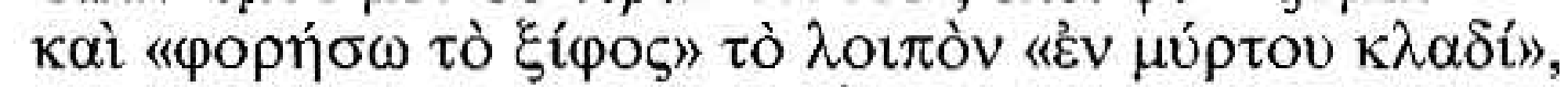

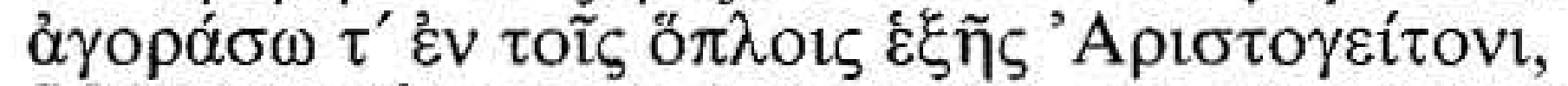

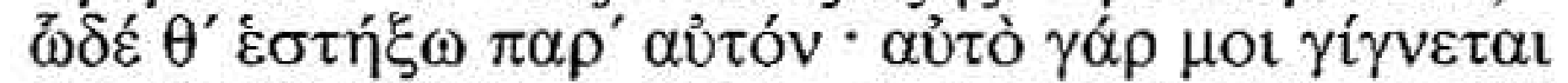

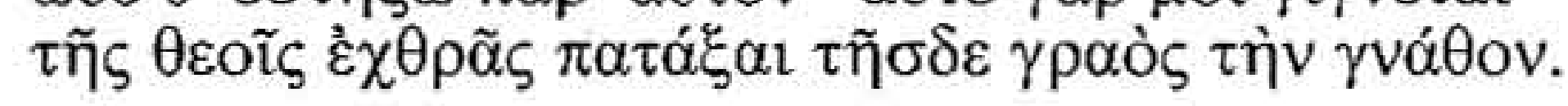

«Oui, voilà qui exhale déjà l'odeur d'affaires plus vastes et plus considérables, ce me semble. Plus que tout je flaire la tyrannie d'Hippias. Et je crains fort que des Laconiens, venus se réunir ici chez Clisthénès $\left({ }^{37}\right)$, n'encouragent ces femmes ennemies des dieux à s'emparer par ruse de nos

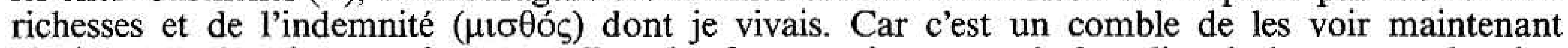
réprimander les citoyens, jacasser, elles, des femmes, à propos de bouclier de bronze et chercher à nous réconcilier avec des Laconiens auxquels on ne peut faire plus confiance qu’à un loup à la gueule béante. Mais, si elles ont tramé cela contre nous, c'est, mes amis, en vue de la tyrannie. Mais moi, elles ne m'imposeront pas leur tyrannie, car je serai sur mes gardes et désormais 'je porterai mon glaive dans un rameau de myrte'. J'irai sur l'Agora en armes à la suite d'Aristogiton, je me tiendrai comme ça près de lui, car me vient justement l'occasion de défoncer la mâchoire de cette vieille haïe des dieux ( $\left.{ }^{38}\right)$ !.

Le chœur fait allusion aux principaux supports de la tradition populaire sur les tyrannoctones, le scolion de l'Harmodios («je porterai mon glaive dans un rameau de myrte») ( ${ }^{39}$ ) et le groupe statuaire des tyrannoctones qui se trouvait sur l'Agora («je me tiendrai comme ça» suggère que le coryphée prend la même pose que la statue d'Harmodios, le bras droit replié au-dessus de la tête, prêt à frapper, et, quand il envisage de se tenir "sur l'Agora... près de lui» (sc. d'Aristogiton), il faut comprendre près de sa statue) $\left({ }^{40}\right)$. Aristophane se moque du culte rendu aux tyrannoctones $\left({ }^{41}\right)$ et s'inscrit dans le même mouvement que Thucydide: de même que

(37) A. H. Sommerstein (Lysistrata, Warminster, 1990, p. 186) note qu'en tant qu'efféminé et homosexuel passif Clisthénès est l'intermédiaire idéal entre les femmes et les Spartiates, réputés enclins à l'homosexualité.

(38) Le coryphée désigne ainsi la coryphée : vieille (cf. 637, 826), haie des dieux (622). Le coup sur la mâchoire est une menace que s'adressent tour à tour les deux chœurs $(657,821)$.

(39) Sur le jeu de mots obscène que contient ce vers, cf. G. LAMBIN, «Dans un rameau de myrte...' (Aristophane, Lys. v. 632)», REG 92, 1979, pp. 542-551.

(40) On trouvera des clichés du groupe statuaire (copie romaine restaurée et conservée au Musée National de Naples) dans S. BRUNNSÂKER, op. cit. (n. 31), pl. 1-3.

(41) La canonisation des tyrannoctones par l'idéologie officielle prit des formes variées d'une ampleur considérable: groupe statuaire sur l'Agora (statues d'Anténor érigées sur l'Agora, mais emportées à Suse par Xerxès ; groupe statuaire de Critios et Nésiotès érigé en 477 et entouré d'un espace réservé); sacrifices annuels offerts par le polémarque (Aristote, Constitution des Athéniens, 58,1); interdiction de donner les noms d'Harmodios et d'Aristogiton à un esclave (AuluGelle, 9,2,10); honneurs dont jouissaient leurs descendants (nourriture au Prytanée...) ; épisème du bouclier d'Athéna figurant les statues des tyrannoctones sur des amphores panathénaïques postérieures à la chute des Trente). Cf. H. 
l'historien affirme que la tyrannie fut renversée à Athènes non par les prétendus tyrannoctones, mais par les Lacédémoniens $\left({ }^{42}\right)$, de même, Lysistrata rappelle aux Athéniens, dans un autre passage de la pièce $\left({ }^{43}\right)$, que ce sont les Spartiates qui les ont libérés de la tyrannie d'Hippias - un fait que les Athéniens préfèrent généralement occulter depuis que Sparte est leur ennemie jurée ( $\left.{ }^{44}\right)$.

Ainsi, dans l'opinion commune athénienne et dans l'idéologie officielle, aspirer à la tyrannie va de pair avec le laconisme ${ }^{(45)}$, et cela à deux titres. Si l'on songe à la tyrannie archaïque, on se souvient que les Spartiates avaient voulu, peu après les réformes de Clisthène, rétablir à Athènes le régime tyrannique et qu'ils avaient mandé Hippias à cet effet $\left.{ }^{46}\right)$. Mais le rapprochement prend aussi son sens dans le cadre d'une identification entre tyrannie et oligarchie, régimes tous deux opposés à la démocratie : le régime spartiate est considéré comme oligarchique et Cléomène avait jadis occupé l'Acropole pour soutenir Isagoras et les oligarques athéniens $\left({ }^{47}\right)$. Du temps même d'Aristophane, Sparte était encore présumée soutenir les oligarques athéniens puisqu'ils firent appel à elle, avec des succès divers, en 411 et en 404 .

\section{c) Oligarchie}

L'image du tyran archaïque interfère, en effet, avec celle des oligarques contemporains, ainsi que l'attestent les sujets d'appréhension invoqués par le chœur des vieillards de Lysistrata:

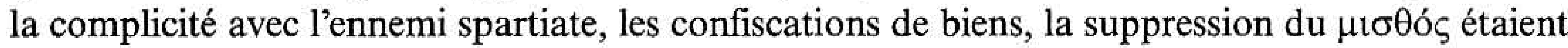
autant de thèmes qui devaient circuler dans Athènes avant même l'abolition de la démocratie par les oligarques.

Tel est donc le faisceau d'images que véhicule le terme de tópavvo s dans l'opinion commune athénienne. C'est sur cette base qu'Aristophane exerce sa satire et qu'il se livre à une véritable démystification des épouvantails populaires et officiels.

\section{LA DÉMYSTIFICATION DES SPECTRES ANTIDÉMOCRATIQUES}

La démystification était l'une des fonctions revendiquées explicitement par le poète lui-même, attaché, selon ses propres termes, à dessiller les yeux de ses concitoyens "gobe-mouches»

Berve, op. cit. (n. 19), I, pp. 73-74, et, pour les sources, II, pp. 562-3. Sur les origines et le sens de ce culte, cf. A. J. Podlecki, «The Political significance of the Athenian 'tyrannicide'-cult», Historia 15, 1966, p. 131 sq.; C. W. FornarA, "The Cult of Harmodius and Aristogeiton», Philologus 114, 1970, pp. 155-180. L'essentiel des témoignages archéologiques et littéraires sur l'image des tyrannoctones est recensé et finement analysé par M. W. TAYLOR, The Tyrant Slayers. The Heroic Image in Fifth Century B.C. Athenian Art and Politics, New York, 1981, qui fait aussi le point des discussions sur le sens politique de ce culte (p. 55 sq.).

(42) VI,53,3: «le peuple savait par la tradition que la tyrannie de Pisistrate et de ses fils était devenue lourde sur sa fin et qu'en outre ce n'était pas lui et Harmodios qui l'avaient renversée, mais bien les Lacédémoniens». Voir, déjà, Hérodote VI,123.

(43) Lysistrata $1150 \mathrm{sq}$.

(44) Malgré Hérodote,Thucydide et Aristophane, bien des Athéniens continuèrent de croire que les tyrans avaient été renversés par Harmodios et Aristogiton. Cf. Platon, Banquet $182 \mathrm{c}$ («c'est l'amour d'Aristogiton et l'amitié affermie d'Harmodios qui brisèrent la domination [des tyrans]») et M. W. TAYLOR, op. cit. (n. 41), n. 6 pp. 189-190.

(45) On peut encore citer le chœur des Guêpes qui traite Bdélycléon à la fois de tyran et de "partisan de Brasidas» (475).

(46) Hérodote, V,91. Cela explique sans doute que le chœur des vieillards de Lysistrata associe directement tyrannie d'Hippias et Laconiens.

(47) Hérodote, V,70, 72. Le chœur des vieillards y fait du reste allusion, toujours dans Lysistrata (274). 
$(\chi \alpha \cup v o \pi \circ \lambda i ́ \tau \alpha \imath)\left({ }^{48}\right)$. On en peut observer de multiples exemples $\left({ }^{49}\right)$, tout particulièrement dans le domaine politique qui nous occupe. Dans le cas présent, elle porte d'abord sur un abus de langage.

\section{A. Túpavvos, un terme galvaudé}

Dans les Guêpes, le chœur harcèle en ces termes Bdélycléon afin qu'il libère son père (484507) :

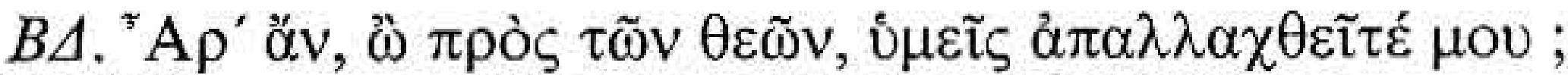

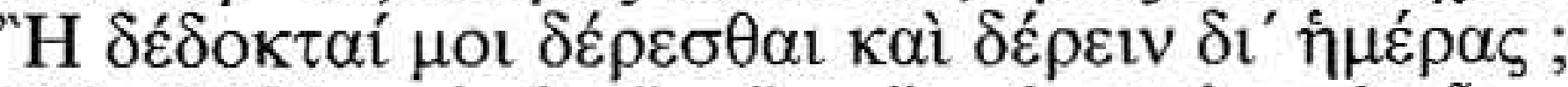

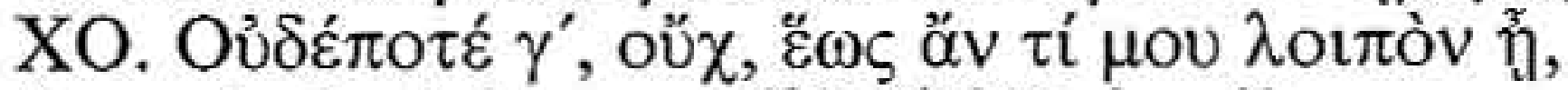

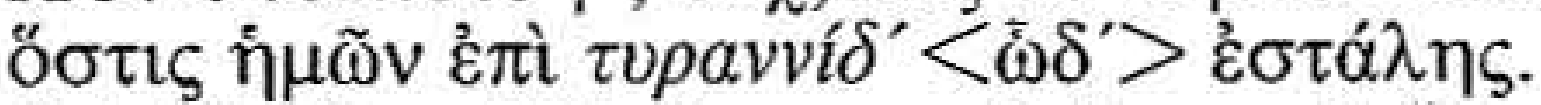

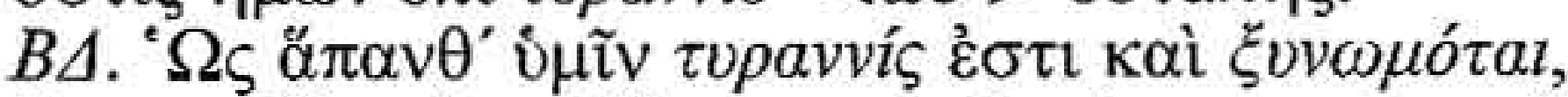

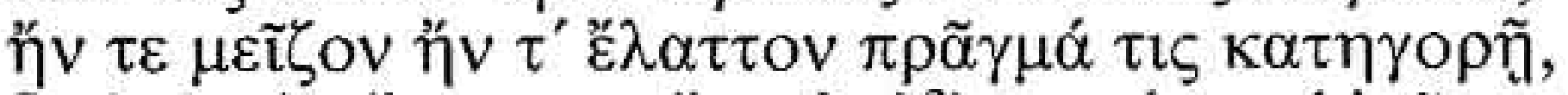

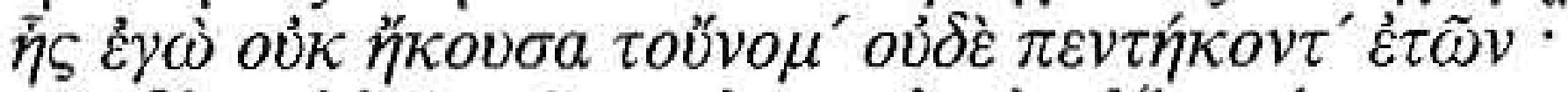

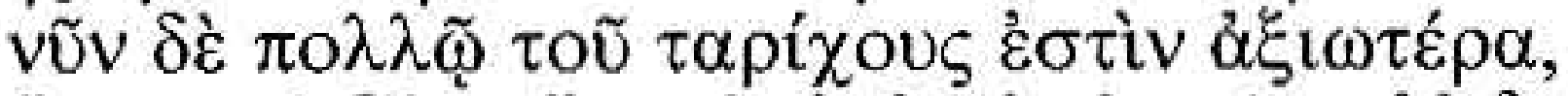

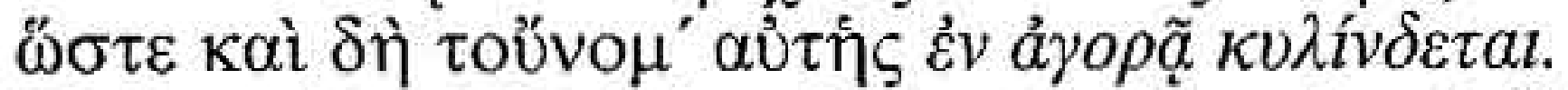

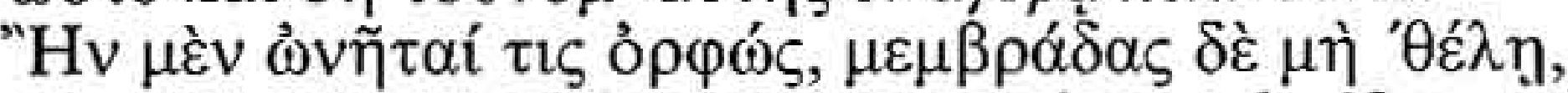

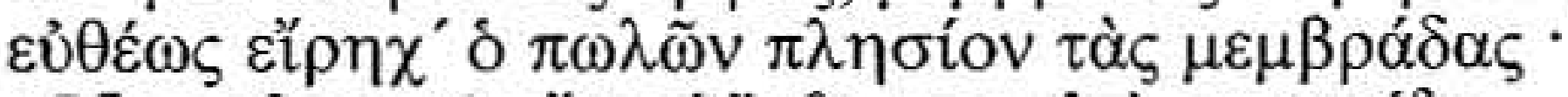

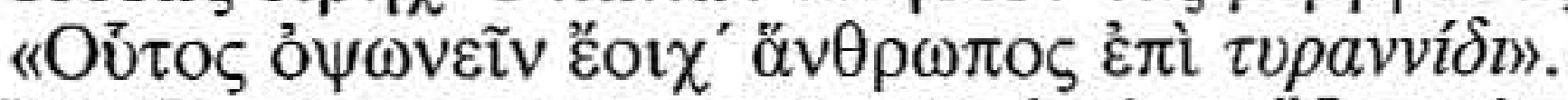

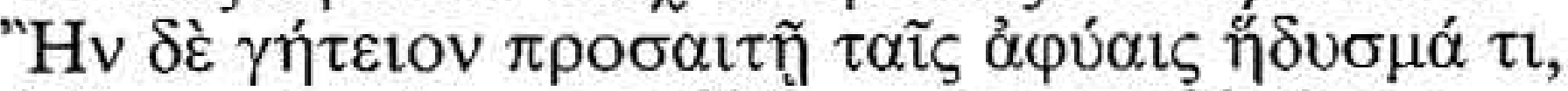

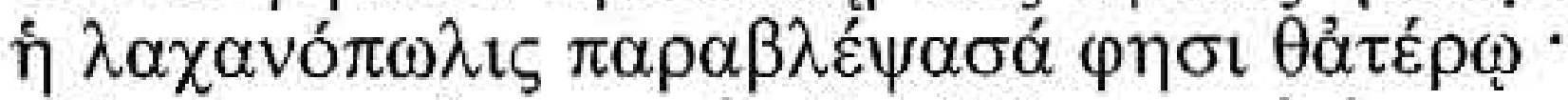

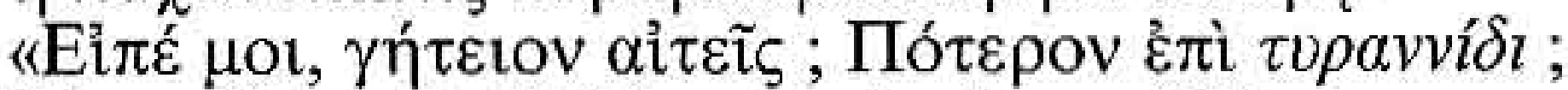

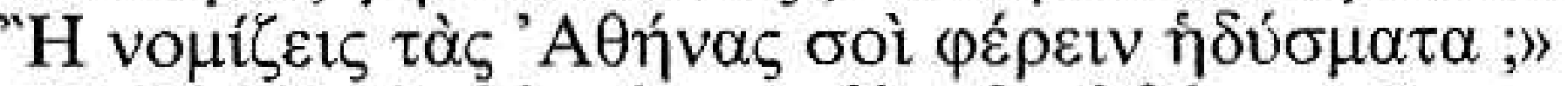

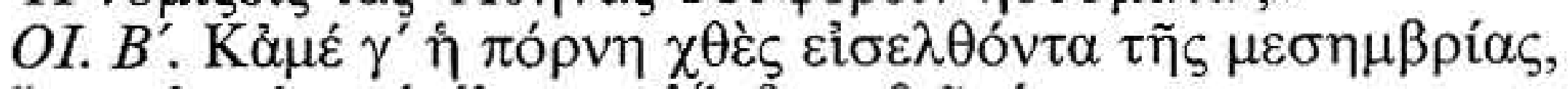

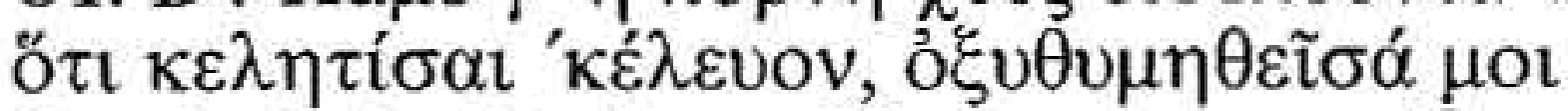

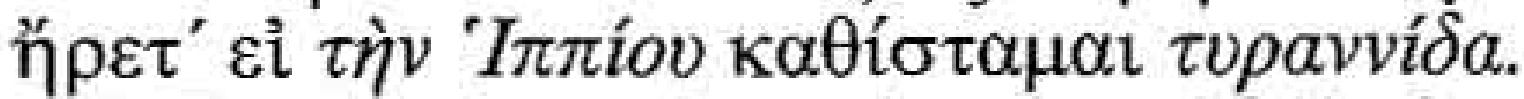

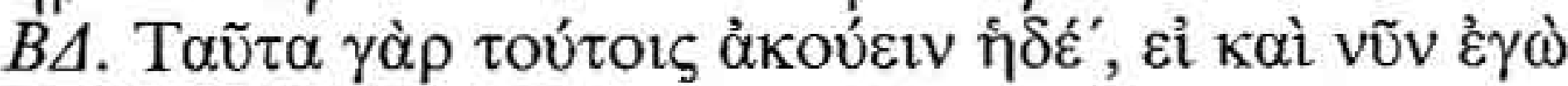

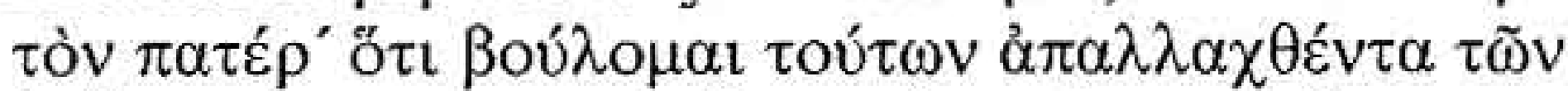

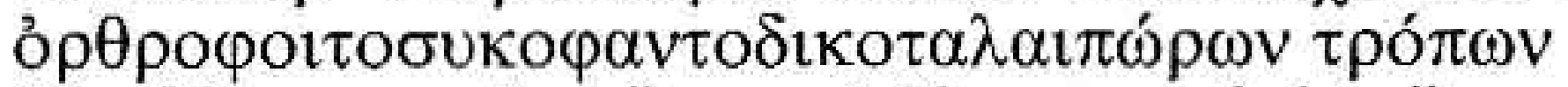

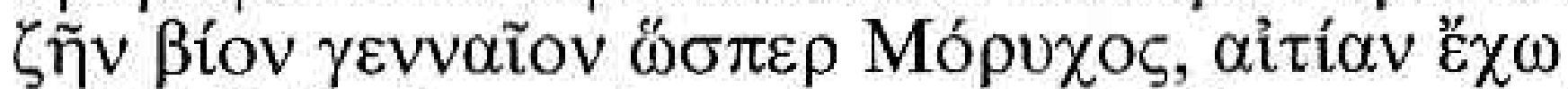

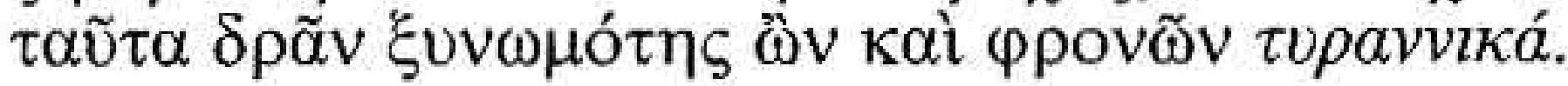

«Bd. - Au nom des dieux, voulez-vous bien me débarrasser le plancher? Ou suis-je condamné à houspiller et me faire houspiller à longueur de journée ?

Chour - Jamais, non, tant qu'il me restera quelque force! puisque, comme on le voit, tu es venu pour exercer sur nous ta tyrannie.

$B d$. - Comme tout est pour vous tyrannie et conspirateurs, quelle que soit l'importance du grief! Je n'en avais pas entendu le nom depuis cinquante ans. Mais à présent elle est bien meilleur marché que le poisson salé, au point que même son nom circule au marché. Si quelqu'un achète des orphes et refuse des sardines, aussitôt le voisin qui vend des sardines s'exclame : "Cet homme a tout l'air de faire des provisions en vue de la tyrannie». Si l'on demande en sus un poireau pour agrémenter quelque peu ses anchois, la marchande de légumes vous regarde de travers en disant : "Dis donc, tu demandes un poireau ? Est-ce en vue de la tyrannie, ou crois-tu qu'Athènes doive te fournir des condiments?

Second serviteur - Moi aussi, la prostituée chez qui j'entrai hier midi, parce que je lui disais de prendre la position hippique, s'est emportée contre moi en me demandant si je voulais rétablir la tyrannie d'Hippias.

$B d$. - Voilà bien les mots que ces gens aiment à entendre, puisque moi, maintenant, parce que je veux voir mon père débarrassé de ces misérables façons d'aller dès l'aurore dénoncer et juger $\left({ }^{50}\right)$, le voir mener la noble vie d'un Morychos, me voilà accusé de le faire parce que je serais un conspirateur et que j'aurais des visées tyranniques».

(48) Cf. Acharniens 633-635 et 656-659.

(49) E. LEV́Y note ainsi que «l'auteur a bien souligné le caractère dérisoire de cet empire que des miséreux, qu'on maintient à dessein dans la misère, s'imaginent exercer sur l'univers" (op. cit. (n. 20), p. 124).

(50) V.-H. Debidour traduit : «ces misérables manies trottalaurorisantes et chicanodénonciatrices». 
Aristophane ironise et Bdélycléon est ici son porte-parole : l'accusation d'aspirer à la tyrannie est manifestement portée à tort et à travers $\left({ }^{51}\right)$. Les situations évoquées n'ont rien de politique, c'est le moins que l'on puisse dire ! Et le moindre détail qui puisse se rattacher à l'image du tyran se trouve mis à profit : contrainte qu'exerce Bdélycléon sur son père; achat d'un

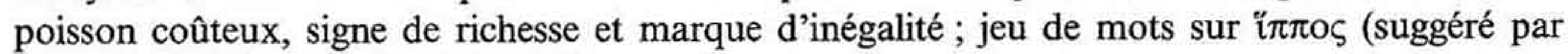
$\kappa \varepsilon \lambda \eta \tau i ́ \sigma \alpha \imath)\left({ }^{52}\right)$ et 'I $\pi \pi i ́ \alpha \varsigma$. Naturellement, les scènes évoquées n'ont aucune prétention réaliste, mais elles caricaturent et amplifient nécessairement une pratique contemporaine qui a galvaudé le terme à force de le faire "circuler au marché». Cela peut rappeler la banalisation de termes français tels que fasciste ou totalitaire, mais ici la soudaineté du phénomène paraît tout à fait frappante.

\section{B. Un reflet de l'actualité politique : menace oligarchique, exploitation démagogique}

Túpavvo prolifère tout particulièrement dans les Guêpes, qui datent de 422 : dans les pièces précédentes, le terme même de tyran n'apparaissait que deux fois et dans des contextes qui ne lui conféraient pas le sens d'une accusation dans la bouche du locuteur ( $\left.{ }^{53}\right)$. Vu le caractère satirique du théâtre d'Aristophane, cette apparition massive reflète manifestement un phénomène survenu depuis peu dans Athènes. A preuve l'observation de Bdélycléon, qui dit n'avoir pas entendu parler de la tyrannie depuis cinquante ans ${ }^{(54)}$.

De même, l'accusation d'être un conspirateur ( $(\xi v \omega \mu$ ót $\eta \varsigma)$ ne figure que dans deux pièces, les Cavaliers et les Guêpes, qui datent respectivement de 424 et de $422\left({ }^{55}\right)$.

$\mathrm{Si}$ l'on ajoute à cela les attaques virulentes dont le túpavvos est la cible dans les Suppliantes d'Euripide, tragédie dont la datation fluctue entre 423 et $420\left({ }^{56}\right)$, on est conduit à penser que ces pièces reflètent un même phénomène, que l'on peut ainsi dater des années 423-422 $\left({ }^{57}\right)$.

Certes, le terme de «tyran» n'a pas cessé d'être employé. Il l'était d'abord dans la routine de la vie civique et politique, puisque l'ostracisme figurait régulièrement à l'ordre du jour de l'Assemblée ( ${ }^{58}$ ) et que, quelle qu'en fût l'utilisation politique, il continua d'être présenté comme une arme défensive contre un tyran potentiel $\left({ }^{59}\right)$. De même, les malédictions à l'adresse des aspirants tyrans, qui semblent avoir ouvert les séances de l'Assemblée et du Conseil $\left({ }^{60}\right)$, ou le culte rendu officiellement aux tyrannoctones $\left({ }^{61}\right)$ assuraient une certaine présence de la figure du tyran dans la cité. En dehors de ce contexte strictement institutionnel, le terme de "tyran»

(51) Voir les passages déjà cités de Guêpes 417 et Lysistrata 616-635 (où le projet réel des femmes, qui est de forcer les hommes à faire la paix, contraste fortement avec l'accusation de tyrannie proférée par les vieillards).

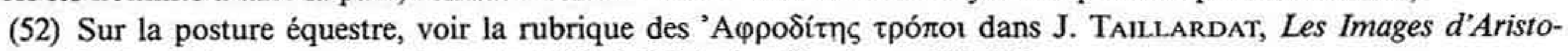

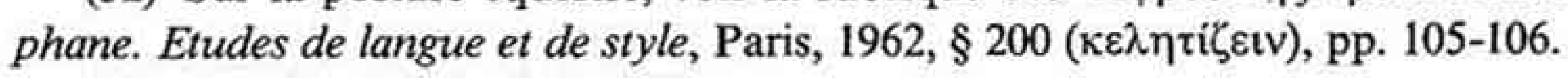

(53) Cavaliers 1114 , Nuées 564.

(54) Guêpes 490.

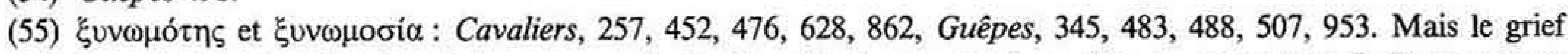
s'exprime aussi dans les Cavaliers à travers une foule de métaphores, sur lesquelles on consultera J. TAILlARDat, p. 232 sq.

(56) Cf. Euripide III, éd. H. GrÉGOIRE, Les Belles Lettres, Paris, 1976, notice pp. 92-98.

(57) En effet, en 424 , les Cavaliers ne reflètent encore aucune hantise de la tyrannie. Cf. E. LÉvy, op. cit. (n. 20), p. 138.

(58) Cf. Aristote, $A P 43,5$.

(59) Cf. V. J. Rosivach, "The Tyrant in Athenian Democracy", Quaderni Urbinati di Cultura classica NS 30 n. 3,1988 , Rome, p. 45 , n. $5,6,7$.

(60) Cf. supra n. 29.

(61) Cf. supra n. 41. 
apparaissait aussi dans des pièces de théâtre qu'une large proportion d'Athéniens pouvait voir : il figure ainsi dans toutes les tragédies conservées d'Euripide (dont la chronologie s'étend approximativement de 438 à la fin du ve siècle av. J.C.), et notamment en 438 (Alceste), en 431 (Médée) et en 428 (Hippolyte) ( ${ }^{62}$ ). De plus, on peut supposer qu'il était «habituel d'accuser un adversaire trop influent d'aspirer à la tyrannie et [que] l'institution même de l'ostracisme ne pouvait qu'inciter à lancer de telles attaques» (63). Mais, dans ces années 420 , il semble que le terme prolifère dans un emploi polémique particulièrement insistant.

Or, l'on sait par Thucydide que le spectre de la tyrannie vint hanter les esprits pendant la guerre-du Péloponnèse. L'historien évoque le souvenir de la tyrannie archaïque pour expliquer les soupçons que l'on entretient à l'égard d'Alcibiade après l'affaire des Mystères (VI,53) et il rend compte de la crainte populaire d'une révolution antidémocratique ("oligarchique ou tyrannique») ( ${ }^{64}$ ). Mais il ne croit guère que la menace ait été sérieuse en 415 (et il disposait pourtant d'un recul dont ne pouvait jouir Aristophane) : il considère que nul ne saura jamais la vérité sur l'affaire des Hermès (VI,60,2) et met en cause à la fois des dénonciateurs sans scrupules et une foule naïve et désemparée $\left({ }^{65}\right)$.

Aristophane n'ajoute pas plus de crédit à la réalité du danger, ni pour les années 420 (comme le montrent les Guêpes en 422), ni après 415 (comme en témoignent les Oiseaux en 414). Il met en cause des hommes politiques qui exploitent l'épouvantail de la conspiration, et notamment Cléon, dans les années $420\left({ }^{66}\right)$. Et, même après 415 , il continue de railler l'épouvantail du tyran, comme dans ce passage des Oiseaux (1071-1075), comédie représentée aux Grandes Dionysies de 414, alors qu'Athènes était encore sous le coup de l'affaire des Hermès $\left({ }^{67}\right)$ :

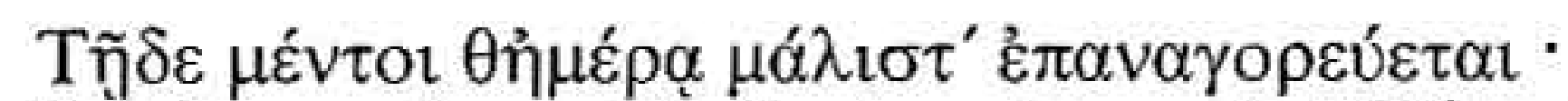

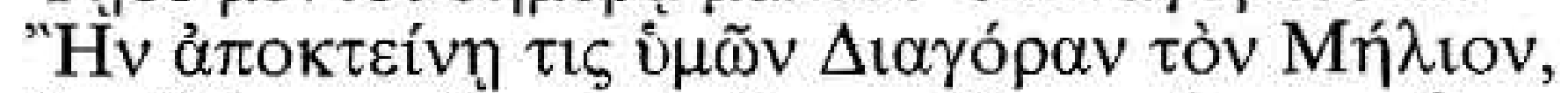

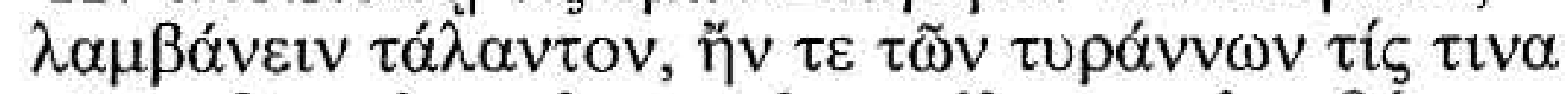
$\tau \tilde{\omega} \nu \tau \varepsilon \theta v \eta \kappa \tau^{\prime} \omega \nu$ ảं

«En ce jour plus que jamais l'on proclame: 'Si l'un d'entre vous tue Diagoras de Mélos, qu'il reçoive un talent, et, s'il tue l'un des tyrans... déjà morts, qu'il reçoive un talent'».

La prime au meurtre fait peut-être allusion à un décret ancien qui mettait à prix la tête des Pisistratides. Elle parodie en tout cas la réaction officielle d'Athènes après la découverte des sacrilèges de 415 : le peuple avait alors offert une prime à qui tuerait les personnes dénoncées dans l'affaire des Hermès ${ }^{\left({ }^{8}\right)}$. Quant à Diagoras de Mélos, philosophe athée notoire, il s'était

(62) Cf. D. Lenfant, art. cit. (n. 2).

(63) E. LEVY, op. cit. (n. 20), p. 138.

(64) VI,60: le peuple athénien «voyait dans tout ce qui s'était passé [Affaires des Hermès et des Mystères] l'indice d'une conjuration visant à l'établissement de l'oligarchie ou de la tyrannie» (trad. D. Roussel).

(65) VI,53 : les Athéniens «sans prendre en considération les antécédents des dénonciateurs et prompts à soupçonner, prêtaient l'oreille à tous les dires. C'est ainsi que, sur la foi de gens sans aveu, ils arrêtèrent et jetèrent en prison des citoyens parfaitement estimables».

(66) La caricature culmine dans les Cavaliers : le Paphlagonien est présenté comme un calomniateur (45,103, 288, $486,491)$, il parle sans cesse de dénoncer $(278,300,445,475-79 \ldots)$, traite les autres de $\xi u v \omega \mu o \tau \alpha i ́ ~(257,452,628$, $862)$ et flaire partout le complot $(314,476 \ldots)$.

(67) Contre l'interprétation des Oiseaux comme pièce de pure fantaisie détachée de l'actualité, voir O. AURENCHE, Les groupes d'Alcibiade, de Léogoras et de Teucros, Paris, 1974, p. 94, qui signale des allusions aux affaires de 415, et E. LÉvy, op. cit. (n. 20), p. 125 n. 2.

(68) Thucydide VI,60,4. 
moqué des Mystères d'Eleusis sans hésiter à en révéler les secrets et l'on sait que sa tête fut également mise à prix $\left({ }^{69}\right)$, sans doute en $415-414\left({ }^{70}\right)$.

Rire d'une peur récente peut certes contribuer à la surmonter, mais il est visible que, là encore, Aristophane tourne en dérision la crainte officielle du tyran. En parlant de tuer des tyrans morts, il fait mine de réduire la menace au retour d'Hippias. Il est clair que, de son point de vue, les tyrans, réels ou potentiels, sont morts depuis longtemps et que le danger est illusoire.

Cette interprétation paraît confirmée par les pièces postérieures à 411. Les dernières véritables

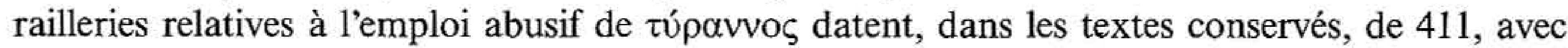
Lysistrata. Cette dernière pièce fut vraisemblablement représentée aux Lénéennes (janvier 411), donc avant la révolution oligarchique. Mais les Thesmophories, sans doute jouées aux Grandes Dionysies de la même année, soit à la fin de mars 411 ( $\left.{ }^{71}\right)$, se ressentent sans nul doute du climat de terreur qui entoura l'instauration du régime des Quatre-Cents $\left({ }^{72}\right)$. Il se peut qu'Aristophane ait choisi la prudence ou qu'il ait cessé de juger chimérique la crainte d'une révolution antidémocratique. Toujours est-il qu'il renonce à toute plaisanterie relative à des tyrans fictifs $\left({ }^{73}\right)$. De même, si, comme on s'en étonne parfois $\left({ }^{74}\right)$, les Thesmophories ne font aucune allusion à la paix, alors que c'était le thème central de Lysistrata, n'est-ce pas qu'entre temps cette paix est devenue un possible slogan des oligarques $\left({ }^{75}\right)$ ?

Par la suite, dans les Grenouilles (405), l'Assemblée des femmes (392) et le Ploutos (388), Aristophane ne reprend pas ses railleries sur l'abus de l'épouvantail tyrannique ( $\left.{ }^{76}\right)$. La nécessité

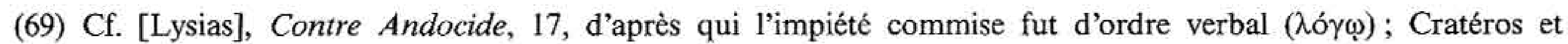
Mélanthios (cités par les scholies aux Oiseaux d'Aristophane 1073), selon qui la tête de Diagoras fut mise à prix parce qu'il dépréciait et divulguait les Mystères.

(70) Cf. L. Woodbury, «The date and atheism of Diagoras of Melos», Phoenix 19, 1965, pp. 178-211. Pour les témoignages et fragments, cf. Diagoras Melius. Theodorus Cyrenaeus, ed. M. WiniarczyK, Teubner, 1981, à compléter avec M. Winiarczyk, «Ergänzungen zu Diagoras und Theodoros», Philologus 133, 1989, p. 151-2.

(71) Sur la datation des deux pièces, cf. A. H. Sommerstein, "Aristophanes and the Events of 411», JHS 97, 1977, pp. 112-126, et T. K. HubBard, The Mask of Comedy, Ithaque-New York, 1991, pp. 243-245.

(72) Sur ce climat de terreur, cf. Thucydide, VIII, 65-66. Sur son reflet dans les Thesmophories, cf. M. CASEvirz, "La politique dans les Thesmophories d'Aristophane: à propos du vocabulaire», in A. Moreau (éd.), Panorama du théâtre antique. Cahiers du GITA n ${ }^{\circ}$ 9, 1996, pp. 93-101, notamment pp. 95-98. D. M. MAcDowell, Aristophanes and Athens, Oxford, 1995, tend au contraire à minimiser la part des allusions politiques dans les Thesmophories, «one of the least political plays» (pp. 251-252).

(73) Les vers 335 sq. et 1143-4 (cités supra) se présentent comme la parodie de formules officielles. SOMMERSTEIN, art. cit., p. 122, y voit l'expression d'une crainte réelle de la tyrannie - et de la tyrannie proprement dite (autrement dit, d'un régime de pouvoir personnel), avec Alcibiade pour acteur (il suit sur ce point T. GeLZER, R.E. Supplementband XII, s.v. Aristophanes (12) (Nachtrag) col. 1468). Thucydide atteste que de tels soupçons touchèrent effectivement Alcibiade (par ex. VI, 15), même si l'ensemble des sources montre que ces insinuations n'ont jamais reposé sur le constat d'un projet politique, mais sur l'examen du comportement d'Alcibiade dans sa vie privée (cf. R. SEAGER, "Alcibiades and the charge of aiming at tyranny», Historia 16, 1967, pp. 6-18). Quoi qu'il en soit, les nombreuses volte-face du personnage et la complexité du climat politique de 411 laissent à penser qu'au moment où les Thesmophories furent représentées, l'existence de la menace antidémocratique était claire, mais que des incertitudes pouvaient peser sur sa nature exacte.

La subtilité du texte d'Aristophane n'était-elle pas précisément de permettre aux spectateurs les deux interprétations (parodie usuelle et anodine, ou avertissement solennel)?

(74) Cf. H. Van Daele, Aristophane IV, Paris, C.U.F., $1991^{8}$, p. 9.

(75) Certes, les oligarques envisagent d'abord de continuer la guerre (cf. Thucydide VIII,63.4) et ce n'est qu'ensuite qu'ils tentent d'engager des négociations avec Agis (VIII,70-71). Mais le soupçon d'une possible connivence entre oligarques et Spartiates a pu naître dès avant cela.

(76) Le seul terme de la famille qui apparaisse dans ces dernières pièces conservées est celui de tupavvíc, qui vise à désigner la souveraineté de Zeus dans un emploi qui n’a rien de polémique (Ploutos 124). 
de renouveler les plaisanteries n'explique pas seule une telle disparition : le sycophante demeure, par exemple, une bête noire indéfectible $\left({ }^{77}\right)$. Il est en fait probable qu'après les expériences oligarchiques de telles moqueries n'aient plus donné matière à rire.

Il semble donc que les affaires de 415 n'aient pas été perçues par tous comme l'expression d'une menace oligarchique sérieuse. A preuve les réserves de Thucydide, qui pourtant connaissait, au moment d'écrire, les événements ultérieurs, et surtout les railleries d'Aristophane, qui supposent l'adhésion d'une bonne partie du public, alors qu'elles disparaissent après 411 . Un tel scepticisme dut être renforcé chez les deux auteurs par leur commun mépris vis-àvis des démagogues pourfendeurs de tyrans imaginaires. L'exagération continue de la menace antidémocratique dans les années 420 et suivantes contribue sans aucun doute à expliquer qu'on ne l'ait pas vue venir en 411, quand elle devint réelle.

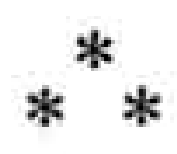

Ainsi, les emplois de $\tau u ́ p \alpha v v o \zeta$ et de $\beta \alpha \sigma \imath \lambda \varepsilon u ́ \varsigma$ se distinguent nettement, chez Aristophane, selon que ces mots se réfèrent à des personnages mythiques ou contemporains. Dans la sphère du mythe, les deux termes sont généralement interchangeables, comme il est fréquent chez Euripide - et cela reflète leur usage tragique. Au contraire, la référence au monde contemporain conduit à adopter l'usage quotidien de ces termes, qui avaient des champs d'application bien distincts. Le $\beta \alpha \sigma \imath \lambda \varepsilon v ́ \varsigma$ est alors un souverain éloigné dans l'espace, sans prétention à régner sur Athènes, alors que túpavvo s ne désigne pas tant le détenteur d'un pouvoir personnel que l'ennemi de la démocratie athénienne, volontiers associé à ce titre à d'autres formes de trahison. Ainsi, Euripide et Aristophane témoignent, chacun à leur manière, de l'évolution qui affecte le terme de $\tau u ́ \rho a v v o \zeta$ au ve siècle, de ses connotations négatives - cependant moins systématiques chez Euripide - et de sa banalisation $\left(^{78}\right)$.

Cette banalisation tourna même au galvaudage et fournit à ce titre un premier ressort comique relevant de la satire sociale et politique : c'est à cette fin qu'Aristophane exagère un abus de langage jusqu'à en exhiber le ridicule. Mais le túpavvo donnait matière à un autre type de plaisanterie couramment pratiqué par l'auteur comique et consistant à prendre un terme au pied de la lettre. De même que, dans les Acharniens, l'CEil du Roi, dont le nom était supposé transcrire un titre officiel à la cour perse, portait sur son masque un oeil gigantesque $\left({ }^{79}\right)$ ou que, dans les Oiseaux, Pisthétaire suivait au pied de la lettre l'expression "aller aux corbeaux»

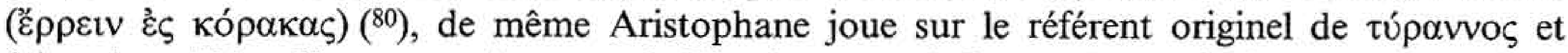

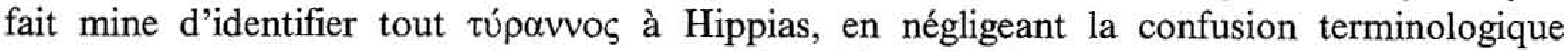
courante entre menace tyrannique et danger oligarchique. Mais le comique se double à plusieurs égards d'une véritable démystification : celle des mythiques tyrannoctones d'abord, mais aussi celle du danger tyrannique à l'époque contemporaine. L'imprécation des femmes des Thes-

(77) Le sycophante est ainsi présent dans presque toutes les pièces conservées et notamment dans l'Assemblée des femmes et le Ploutos (il ne figure pas dans Nuées, Lysistrata, Grenouilles), mais apparait dans les Thesmophories (529 sq.) à travers la métaphore de celui qui mord (Taillardat $§ 727$ p. 424).

(78) Chez Euripide, le terme est devenu plus fréquent que $\beta \alpha \sigma \iota \lambda \varepsilon v ́ \varsigma$ et Aristophane témoigne, quant à lui, de l'usage généralisé qu'en font les Athéniens. De plus, la famille du mot s'est agrandie depuis Hérodote. Ce dernier

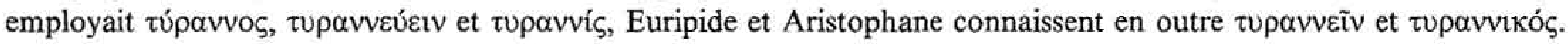

(79) Acharniens 94 sq.

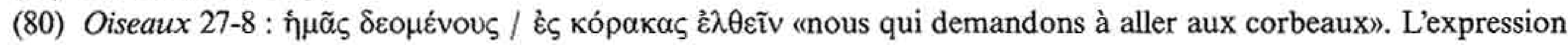
équivaut à peu près au français «aller au diable». 
mophories contre ceux qui voudraient «aider au retour du tyran» $\left({ }^{81}\right)$ reproduit sans doute une formule officielle héritée de l'époque où Hippias pouvait encore revenir au pouvoir avec l'appui des Perses et de ses partisans athéniens, quelque quatre-vingts ans plus tôt. La mise à prix de la tête des «tyrans morts» dans les Oiseaux dénonce encore plus clairement ce décalage comique. Mais ce qu'Aristophane entend également attaquer, ce sont les accusations de conspiration dont abusent les démagogues et les attitudes ridicules qu'elles font naitre. Il semble bien que, pas plus que Thucydide, l'auteur comique n'ait cru que se dissimulait derrière les affaires de 415 une conspiration oligarchique ou tyrannique. Mais, après 411 , il renonça à faire rire de menaces antidémocratiques qui s'étaient révélées bien fondées.

Dominique Lenfant (Strasbourg)

(81) Thesmophories 339. Cf. supra p. 191. 